OPEN ACCESS

Edited by:

Christian Lohr,

University of Hamburg, Germany

Reviewed by:

Gustavo Alberto Chiabrando, National University of Córdoba,

Argentina

Kyung Seok Han

Dongguk University Gyeongju,

South Korea

*Correspondence:

Andreas Faissner

andreas.faissner@rub.de

Specialty section:

This article was submitted to

Non-Neuronal Cells,

a section of the journal

Frontiers in Cellular Neuroscience

Received: 16 December 2020

Accepted: 26 January 2021

Published: 18 February 2021

Citation:

Romeo R, Boden-El Mourabit $D$, Scheller A, Mark MD and Faissner $A$

(2021) Low-Density Lipoprotein Receptor-Related Protein 1 (LRP1) as a Novel Regulator of Early Astroglial

Differentiation.

Front. Cell. Neurosci. 15:642521.

doi: 10.3389/fncel.2021.642521

\section{Low-Density Lipoprotein} Receptor-Related Protein 1 (LRP1) as a Novel Regulator of Early Astroglial Differentiation

\author{
Ramona Romeo ${ }^{1}$, Damian Boden-El Mourabit ${ }^{2}$, Anja Scheller ${ }^{3}$, Melanie D. Mark² and \\ Andreas Faissner ${ }^{1 *}$ \\ ${ }^{1}$ Department of Cell Morphology and Molecular Neurobiology, Ruhr-University Bochum, Bochum, Germany, ${ }^{2}$ Behavioral \\ Neuroscience, Ruhr-University Bochum, Bochum, Germany, ${ }^{3}$ Department of Molecular Physiology, Center for Integrative \\ Physiology and Molecular Medicine (CIPMM), University of Saarland, Homburg, Germany
}

Astrocytes are the most abundant cell type within the central nervous system (CNS) with various functions. Furthermore, astrocytes show a regional and developmental heterogeneity traceable with specific markers. In this study, the influence of the lowdensity lipoprotein receptor-related protein 1 (LRP1) on astrocytic maturation within the hippocampus was analyzed during development. Previous studies mostly focused on the involvement of LRP1 in the neuronal compartment, where the deletion caused hyperactivity and motor dysfunctions in knockout animals. However, the influence of LRP1 on glia cells is less intensively investigated. Therefore, we used a newly generated mouse model, where LRP1 is specifically deleted from GLAST-positive astrocytes colocalized with the expression of the reporter tdTomato to visualize recombination and knockout events in vivo. The influence of LRP1 on the maturation of hippocampal astrocytes was assessed with immunohistochemical stainings against stage-specific markers as well as on mRNA level with RT-PCR analysis. The examination revealed that the knockout induction caused a significantly decreased number of mature astrocytes at an early developmental timepoint compared to control animals. Additionally, the delayed maturation of astrocytes also caused a reduced activity of neurons within the hippocampus. As previous studies showed that the glial specification and maturation of astrocytes is dependent on the signaling cascades Ras/Raf/MEK/Erk and PI3K/Akt, the phosphorylation of the signaling molecules Erk1/2 and Akt was analyzed. The hippocampal tissue of LRP1-deficient animals at P21 showed a significantly decreased amount of activated Erk in comparison to control tissue leading to the conclusion that the activation of this signaling cascade is dependent on LRP1 in astrocytes, which in turn is necessary for proper maturation of astrocytes. Our results showed that the deletion of LRP1 at an early developmental timepoint caused a delayed maturation of astrocytes in the hippocampus based on an altered activation of the Ras/Raf/MEK/Erk signaling 
pathway. However, with ongoing development these effects were compensated and the number of mature astrocytes was comparable as well as the activity of neurons. Therefore, LRP1 acts as an early regulator of the differentiation and maturation of astrocytes within the hippocampus.

Keywords: astrocyte heterogeneity, differentiation, astrocyte functions, hippocampus, in vivo knockout model, LRP1

\section{INTRODUCTION}

Astrocytes are the most abundant cell type within the central nervous system (CNS) and were formerly known as passive players supplying neurons with energy substrates and providing structural support (Yong et al., 1998; Gordon et al., 2007; Iadecola and Nedergaard, 2007; Sofroniew and Vinters, 2010). However, more recent studies prove that astrocytes are actively contributing to the neuronal transmission in forming the tripartite synapse with pre- and postsynapse (Bushong et al., 2004; Freeman, 2010; Savtchouk and Volterra, 2018). The majority of astrocytes are generated postnatally after the generation of neurons (Kriegstein and Alvarez-Buylla, 2009). The postnatal and terminal differentiation into astrocytes seem to be dependent on intracellular signaling cues (Qian et al., 2000; Shen et al., 2006). Previous studies have shown that the glial specification is influenced by the activation of the Ras/Raf/MEK/Erk pathway (Franzdóttir et al., 2009; Li et al., 2012). The activity of these signaling pathways is regulated by the formation of cell surface receptor complexes causing intracellular signaling cascades. One known activator of the Ras/Raf/MEK/Erk pathway is the complex formation between the low-density lipoprotein receptor-related protein 1 (LRP1) with the plateletderived growth factor receptor $\beta$ (PDGFR $\beta$ ) (Boucher et al., 2002; Loukinova et al., 2002) or tropomyosin receptor kinase A (trkA) (Spuch et al., 2012). Furthermore, the association of LRP1 with PDGFR $\beta$ also influences the activation of the PI3K/Akt pathway, another important signaling cascade involved in glia specification (Muratoglu et al., 2010).

LRP1 is a member of the low-density lipoprotein receptor (LDL) superfamily and is also known as CD91 or $\alpha 2$ macroglobulin receptor (Binder et al., 2000; Liu et al., 2000; Marschang et al., 2004). The receptor is ubiquitously expressed but enriched in liver, blood vessels and the CNS (Lillis et al., 2005). The receptor consists of an extracellular, $515 \mathrm{kDa} \alpha$-chain and an intracellular, $85 \mathrm{kDa} \beta$-chain, non-covalently bound on the cell surface (Lillis et al., 2005). Over 40 potential ligands are known for LRP1 and ligand binding to LRP1 leads to endocytosis of the whole complex, making LRP1 formerly known for its endocytic function (Lillis et al., 2005; Bres and Faissner, 2019). In addition, LRP1 can form complexes with other receptors leading to the activation of intracellular pathways. Thereby, LRP1 was unraveled as a regulator for different cellular processes, such as proliferation (Zhang and Liu, 2002), differentiation (Seger and Krebs, 1995; Ortega and Alcántara, 2010; Safina et al., 2016) and apoptosis (Burris, 2013; Kumar et al., 2013). Total loss of LRP1 within the whole organism leads to embryonic lethality, highlighting the importance of LRP1 (Herz et al., 1992, 1993).
However, the influence of LRP1 in regard to astrocyte function was not intensively studied. The deletion of LRP1 in astrocytes caused a negatively influenced cellular uptake of $A \beta$ and degradation involved in Alzheimer's Disease (AD) progression (Liu et al., 2017). The deletion of LRP1 from radial glia cells resulted in altered numbers of astroglial subpopulations in vivo (Bres et al., 2020). Furthermore, we investigated the influence of LRP1 on astrocytes in vitro, where we observed that the knockout of LRP1 in primary cortical astrocytes caused an altered expression of mature astrocytic markers. Additionally, the functionality of astrocytes in regard to the neuronal transmission as well as on synaptogenesis of hippocampal neurons was negatively influenced (Romeo et al., 2021).

Based on the findings and preliminary data we decided to investigate the influence of LRP1 on the maturation and functions of hippocampal astrocytes in vivo. Therefore, we used our newly generated mouse model (Romeo et al., 2021), where LRP1 is specifically deleted from GLAST-positive astrocytes via the intraperitoneal injection of Tamoxifen into lactating mothers causing the loss of LRP1 in their litters. Via the expression of the reporter tdTomato, we were able to visualize recombination events as well as the deletion of LRP1. The maturation of astrocytes in LRP1-deficient tissue was assessed via immunohistochemical stainings as well as with RT-PCR. Additionally, we wanted to further characterize our knockout model and performed behavioral tests to investigate the motor coordination and cognitive abilities.

\section{MATERIALS AND METHODS}

\section{Animals}

As previously described (Romeo et al., 2021), we generated a new triple transgenic mouse model where $\operatorname{Lrp} 1$ was specifically deleted from GLAST-positive astroglial precursor cells by using the GLAST::CreERT2-mouse line (Mori et al., 2006) bred with the LRP1 $1^{\text {flox/flox}}$-mouse line (Rohlmann et al., 1996). For the mating of control, respectively, knockout animals, animals with a heterozygous expression of the Cre recombinase under the GLAST promotor were used (Romeo et al., 2021). Therefore, not all pups of the litters were suitable for the study as some expressed the Cre recombinase homozygously or not at all. Additionally, the application of Tamoxifen to lactating mothers caused mortality of the pups. This study was performed in approval with the State Agency for Nature, Environment and Consumer Protection Northrhine-Westphalia (Landesamt fuer Umweltschutz, Naturschutz und Verbraucherschutz; file number: 84-02.04.2016.A482). The animals were housed with a 
$12 \mathrm{~h}$ light/dark cycle and access to food and water ad libitum. To induce the knockout in the new born pups lactating mothers received $100 \mathrm{mg} / \mathrm{kg}$ of bodyweight of a $10 \mathrm{mg} / \mathrm{ml}$ stock solution of Tamoxifen (TAM; Sigma Aldrich, Chemie GmbH, Munich, Germany, Catalog-No.: t5648) in corn oil 5 days after birth. TAM was applied for 5 days in a row in accordance to Jahn et al. (2018). The genotyping of the animals was performed as described in Romeo et al. (2021). Briefly, tail biopsies were taken and lysed with $200 \mu \mathrm{l}$ of DirectPCR ${ }^{\circledR}$ Lysis Reagent Tail (Peqlab, VWR Life Science, Radnor, PA) and $0.2 \mathrm{mg} / \mathrm{ml}$ Proteinase-K (Sigma-Aldrich, Chemie $\mathrm{GmbH}$, Munich, Germany) at $55^{\circ} \mathrm{C}$ at $350 \mathrm{rpm}$ overnight. The lysis was stopped by heating the samples to $85^{\circ} \mathrm{C}$ for $45 \mathrm{~min}$ at $350 \mathrm{rpm}$. For the genotyping, gene-specific primers were used.

\section{Transcardial Perfusion of Animals}

For the investigation of the influence of LRP1 on the maturation of astrocytes and their functionality, three developmental stages were analyzed (P21, P28, and P56-P70, summarized as adult). At the stage-specific age either control or knockout animals were anesthetized with $100 \mathrm{mg} / \mathrm{kg}$ ketamine (CPPharma, Burgdorf, Germany), $10 \mathrm{mg} / \mathrm{kg}$ Xylazin (CP-Pharma) in $0.9 \% \mathrm{NaCl}$. Afterward, the animals were transcardially perfused with phosphate buffered saline (PBS; $137 \mathrm{mM}$ sodium chloride, $3 \mathrm{mM}$ potassium chloride, $6.5 \mathrm{mM}$ disodium hydrogen phosphate, $1.5 \mathrm{mM}$ potassium dihydrogen phosphate; $\mathrm{pH} 7.3$ ) and subsequently with $4 \%(\mathrm{w} / \mathrm{v})$ paraformaldehyde (PFA) when the tissue was used for immunohistochemistry. Tissue for the RNA and protein isolation was prepared and transferred to a dish containing PBS. Then the hippocampus was removed and frozen in liquid nitrogen until further use.

\section{Immunohistochemistry}

Prepared tissue was post-fixed in 4\% (w/v) PFA for additional $6 \mathrm{~h}$ and then dehydrated in a sucrose gradient (10\%, 20\%, 30\% $(\mathrm{w} / \mathrm{v})$ sucrose in PBS). Afterward, the tissue was embedded in Tissue Freezing Medium (Leica, Wetzlar, Germany) at $-80^{\circ} \mathrm{C}$ until further use. Cryosections of $14 \mu \mathrm{m}$ were prepared. The cryosections were stained as previously described (Schäfer et al., 2019; Ulc et al., 2019). Shortly, cryosections were transferred to citric acid buffer (solution A: $0.1 \mathrm{M}$ citric acid-1-hydrate, solution B: $0.1 \mathrm{M} \mathrm{Na-citrate-dihydrate;} 1 \mathrm{mM}$ of solution A and $4 \mathrm{mM}$ of solution $\mathrm{B}$ in aqua dest.) for $1 \mathrm{~h}$ at $70^{\circ} \mathrm{C}$. After cooling and blocking $(10 \%(\mathrm{v} / \mathrm{v})$ normal goat serum (NGS; Jackson Immuno Research Labs, Catalog-No.: 005-00-121; AB_2336990) in PBT-1 [10\% (v/v) BSA, 0.1\% (v/v) Triton-X 100 in $\mathrm{PBS})$ ] for $1 \mathrm{~h}$, primary antibodies were diluted in blocking solution and sections were incubated over night at $4^{\circ} \mathrm{C}$. For our analysis we used the following primary antibodies: LRP1 (1:500, Abcam, Catalog-No.: ab92544; AB_2234877), GFAP (1:300, DAKO, Catalog-No.: Z0334; AB_10013382), GLT-1 (EAAT2; 1:100, Santa Cruz, Catalog-No.: sc-365634; AB_10844832), S100 (1:750, Sigma-Aldrich, Catalog-No.: ab868; AB_306716), c-Fos (1:500, Abcam, Catalog-No.: ab208942; AB_2747772), CC1 (APC; 1:100, Abcam, Catalog-No.: ab16794; AB_443473), NeuN (1:500, Millipore, Catalog-No: MAB377; AB_2298772) and phospho-Histone H3 (PH3; 1:300, Millipore, Catalog-No.:
06-570; AB_310177). Further on, sections were washed thrice with PBS for $20 \mathrm{~min}$, followed by incubation with appropriate secondary antibodies diluted in PBS/A ( $0.1 \%$ bovine serum albumin (w/v; BSA; Sigma-Aldrich, Catalog-No.: A7030) in PBS) for $2 \mathrm{~h}$ at room temperature. We used the following secondary antibodies coupled with Cy2: Goat anti-mouse Cy2, Jackson Immuno Research Labs, Catalog-No.: 115-545-044; AB_2338844 and Goat anti-rabbit Cy2, Jackson Immuno Research Labs, Catalog-No.: 111-545-045; AB_2338049 (1:250) and the cell nuclei marker Hoechst $(1: 100)$. Further on, the washing steps with PBS were repeated in the dark and the sections were covered with Immu-Mount (Thermo Fisher Scientific, Waltham, Massachusetts, United States; Catalog-No.: 9990402). Images were taken with the AxioZoom by Zeiss (Oberkochen, Germany).

\section{RNA Isolation, cDNA Synthesis and Real-Time PCR Analysis}

One hippocampal half of animals was lysed with lysis buffer of the Gene Elute Mammalian Total RNA Miniprep Kit (Sigma Aldrich; Catalog-No.: RTN350-1KT) and RNA was isolated according to manufacturer's manual. To synthesize cDNA, $1 \mu \mathrm{g}$ of RNA was used in accordance to the First Strand cDNA-synthesis Kit (Fermentas, Waltham, MA, United States; Catalog-No.: K1612). To investigate the maturation of astrocytes within astrocytespecific LRP1-depleted hippocampi, stage-specific astroglial markers were used (see Table 1) with $\beta$-actin as reference gene.

\section{Sample Synthesis and Western Blotting}

The other hippocampal half was lysed with radioimmunoprecipitation assay buffer (RIPA; $10 \mathrm{mM}$ Tris$\mathrm{HCl}$ ( $\mathrm{pH}$ 8.0), $1 \mathrm{mM}$ ethylenediaminetetraacetic acid (EDTA), $0.5 \mathrm{mM}$ ethylene glycol-bis( $\beta$-aminoethyl ether)-N,N,N'N'tetraacetic acid (EGTA), 1\% (v/v) Triton X-100, 0.1\% (w/v) sodium deoxycholate, $0.1 \%(\mathrm{v} / \mathrm{v})$ sodium dodecyl sulphate (SDS), $140 \mathrm{mM} \mathrm{NaCl}$, Sigma) with $1 \%(\mathrm{v} / \mathrm{v})$ of protease inhibitors phenylmethylsulfonyl fluoride (PMSF) and $1 \%(\mathrm{v} / \mathrm{v})$ aprotinin (APR). The tissue was manually lysed and further on triturated with pipettes to dissolve the tissue. Protein concentration was measured with the Pierce ${ }^{\mathrm{TM}} \mathrm{BCA}^{\mathrm{TM}}$ Protein Assay (Thermo Fisher Scientific). For the western blot analysis 30 $\mu \mathrm{g}$ of protein was applied to a $12 \%$ sodium dodecyl sulfate polyacrylamide (SDS) gel as described in Hennen et al. (2011). We used following primary antibodies: LRP1 (1:10,000, Abcam, Catalog-No.: ab92544; AB_2234877), pAkt (1:5,000, Cell Signaling Technologies, Catalog-No.: \#4,060, AB_2315049), tAkt (1:5,000; Cell Signaling Technologies, Catalog-No.: \#4,691, AB_915783), pErk1/2 (1:5,000; Santa Cruz, Catalog-No.: sc7383, AB_627545), tErk1/2 (1:5,000; Santa Cruz, Catalog-No.: sc-514302, AB_2571739), GLT-1 (EAAT2; 1:200, Santa Cruz, Catalog-No.: sc-365634; AB_2571739) and $\alpha$-tubulin (1:5,000, Sigma Aldrich, Catalog-No.: T9026, AB_477593), used as control and for normalization. The protein expression was detected with the Clarity ${ }^{\mathrm{TM}}$ Western ECL Substrate by BioRad (Feldkirchen, Germany) and visualized with the MicroChemie chemiluminescence device with the Gel Capture Software by biostep (Burkhardtsdorf, Germany; Version: 6.6). 
TABLE 1 | Investigated genes in the expression profile analysis of hippocampal tissue of either LRP1-deficient animals compared to control animals in vivo.

\begin{tabular}{|c|c|c|}
\hline Gene & Primer sequence & GenBank no. \\
\hline$\beta$-actin & F: 5'-TATGCCAACACAGTGCTGTCTGGTGG-3' R: 5'-TAGAAGCATTTGCGGTGGACAATGG-3' & NM_007393.5 \\
\hline Akt1 & F: 5'-5'-CGACGTAGCCATTGTGAAGG-3' R: 5'-CTTCCTGCCTCTTGAGTCCA-3' & NM_009652.3 \\
\hline Aldh1/1 & F: 5'-GGAAGTTGAGAGGGGAGGAC-3' R: 5'-GGAAGTTGAGAGGGGAGGAC-3' & BC030730.1 \\
\hline Aquaporin-4 & F: 5'-TTGCTTTGGATCAGCATTG-3' R: 5'-TGAGCTCCACATCAGGACAG-3' & NM_009700.3 \\
\hline Fgfr3 & F: 5'-GTCCTGTTCTGGCCAATGTT-3' R: 5' -GTTCTGGCAGCCAAGTCTC-3' & NM_001205270.1 \\
\hline Gfap & F: 5'-CGACTATCGCCGCCAACTGC-3' R: 5' -GCGATCTCGATGTCCAGGGCT-3' & NM_001131020.1 \\
\hline Glast & F: 5'-GGCGGCCCTAGATAGTAAGG-3' R: 5'-AGAGTCTCCATGGCCTCTGA-3' & XM_021208184.2 \\
\hline Glt-1 & F: 5'-ATGATCATGTGGTACTCCCCTC-3' R: 5'-TTGTCGTCGTAAATGGACTGC-3' & NM_001077514.4 \\
\hline Gria1 & F: 5'-CCGTTGACACATCCAATCAG-3' R: 5'-GTTGGCGAGGATGTAGTGGT-3' & NM_001113325.2 \\
\hline Gria2 & F: 5'-AACGGCGTGTAATCCTTGAC-3' R: 5'-CTCCTGCATITCCTCTCCTG-3' & NM_013540.3 \\
\hline Grin1 & F: 5'-CGGCTCTTGGAAGATACAGC-3' R: 5'-TTGTAGACGCGCATCATCTC-3' & NM_008169.3 \\
\hline Grin2a & F: 5'-GCTGTCAGCACTGAATCCAA-3' R: 5'-ATCCCTGGGAGAACTTGCTT-3' & NM_008170.3 \\
\hline Grin2b & F: 5'-GTGAGAGCTCCTITGCCAAC-3' R: 5'-GGGTTGGACTGGTTCCCTAT-3' & NM_008171.3 \\
\hline Lrp1 & F: 5'-GGTAGTTGTTCCTCAATGCTC-3' R: 5'-TGTTGCTGACTAACAACCTGCT-3' & NM_008512.2 \\
\hline Lrp2 & F: 5'-CTTCTGATGAGTCCGCTTGC-3’ R: 5'-AGTTCCCATTGCTGCACTTG-3' & NM_001081088.2 \\
\hline Mtor & F: 5'-CTTGCTGATCCTCAACGAGC-3' R: 5'-CTGGATCAGCGAGTTCTTGC-3' & NM_020009.2 \\
\hline Nestin & F: 5'-CTCGAGCAGGAAGTGGTAGG-3' R: 5'-GTTAGCGCTGCCRCRAGACC-3' & NM_016701.3 \\
\hline$S 100$ & F: 5'-TGTCTTCCACCAGTACTCCG-3' R: 5'-ACTCCTGGAAGTCACACTCC-3' & NM_009115.3 \\
\hline
\end{tabular}

The used primers were self-designed with the according nucleotide sequence (see GenBank No.).

\section{Motor and Cognitive Behavior Tests}

To investigate if the absence of astroglial LRP1 has an influence on the behavior or motor coordination, we used six conditional knockout animals (GLAST ${ }^{\mathrm{CreERT} 2 / \mathrm{wt}} \mathrm{Rosa} 6^{\mathrm{fl} / \mathrm{fl}} \mathrm{LRP} 1^{\mathrm{fl} / \mathrm{fl}}$; 4 males, 2 females) and five control animals (GLAST ${ }^{\text {CreERT2 } /{ }^{\text {wt }} \text { Rosa26 }}{ }^{\mathrm{fl} / \mathrm{fl}} \mathrm{LRP} 1{ }^{\mathrm{wt} / \mathrm{wt}} ; 4$ males, 1 female) at the age of 2 months, for all tests. The rotarod test assessed the motor coordination as well as the balance of the knockout animals in comparison to the control animals as described (Mark et al., 2011; Maejima et al., 2013). Briefly, after acclimation to the rotarod device (Columbus Instruments) with four rotations per minute (rpm) for $1 \mathrm{~min}$ the rod was accelerated at $0.1 \mathrm{rpm} / \mathrm{s} \mathrm{up}$ to $40 \mathrm{rpm}$. Speed and latency to fall were recorded and averaged from three trials per mouse. To test for fine motor coordination mice were tested on the beam walk. Mice were placed on a horizontally $70 \mathrm{~cm}$ long beam $(1 \mathrm{~cm}$ wide and $60 \mathrm{~cm}$ above the table surface) with a $20 \mathrm{~cm}^{2}$ goal box. The animals underwent 2 days of training (six trials per day) before the data was collected. The time to transverse the beam, idle time (immobile time at start), the number of slips of the right and left hindlimbs and falls were investigated. Animals were given a maximum of $120 \mathrm{~s}$ to reach the goal box or for a fall. The collected data was averaged over three trials per mouse. The muscle strength of the mice was evaluated with the hangwire test. Mice were placed upside down on a wire screen $(1.2 \times 1.2 \mathrm{~cm}) 50 \mathrm{~cm}$ above a cage and the latency to fall was recorded. Another test to investigate motor coordination and balance is the vertical pole test. Here, the mice were placed on a $50 \mathrm{~cm}$ vertical metal pole $(1 \mathrm{~cm}$ diameter) facing upwards. The latency to descend down to the cage was recorded. When the animals fell down or slid down the pole rear end first, the maximum of $120 \mathrm{~s}$ was given. To assess the cognitive abilities that are related to memory and learning processes the 2-object novel object recognition test (NOR) based on the established protocol (Lueptow, 2017) was used. Briefly, the test consisted of 3 days, habituation to the arena $(50 \times 50 \mathrm{~cm})$, testing to two similar objects and testing to one familiar and one novel object. The latency to investigate the new object in comparison to the familiar one was recorded and defined as the preference index. Cognitive functions of the mice were also investigated using the T-maze test (Deacon and Rawlins, 2006). Briefly, mice were placed in a T-shaped arena and explored the left or right arm of the $\mathrm{T}$. The mice chose one arm based on curiosity. When the mouse entered one of the arms in the $\mathrm{T}$ a door at the start of the arm was closed to allow $30 \mathrm{~s}$ exploration. Then the mice were again placed at the start of the arena and were allowed to choose one arm. Unperturbed animals would explore the previously unknown arm of the arena, whereas impaired animals were not able to remember which part was already explored. Five trials per animal were averaged and the alternation value was evaluated.

For the statistical analyses, we firstly evaluated if the data was normally distributed with the Shapiro-Wilk-Normality test. As the data was normally distributed the Student's $t$-test was used to investigate the level of significance $\left({ }^{*} p \leq 0.05,{ }^{* *} p \leq 0.01\right.$, $* * * p \leq 0.001)$.

\section{Statistical Analyses}

The immunohistochemical stainings were investigated with the cell counter plug-in by Image J. Here, all tdTomatopositive cells were quantified in the spaces between the Cornu Ammonis layers of the hippocampus to provide the most accurate quantification of recombined cells (see Supplementary Figure 1). Afterward, all marker-specific and tdTomato-double positive cells were quantified to evaluate specificity of recombination. Exemplary double-positive cells, meaning tdTomato- and marker-positive cells, were highlighted with arrowheads in the figures. Additionally, the nuclei within the molecular layers 
of the hippocampus were evaluated to investigate the number of recombined cells. Intensity of the bands of the PCR and western blot analyzes were also measured with ImageJ. The band intensities were normalized to the expression of $\beta$-actin, respectively, $\alpha$-tubulin. The results are shown as the mean \pm the standard error of the mean (SEM). The endpoint-PCR analysis was used as a semi-quantitative method to analyze potentially differentially expressed genes after knockout indication. The cycle number for the RT-PCR were below the amplificate saturation level. The depicted PCR results are only shown as an example. For the statistical analyses, we firstly evaluated if the data was normally distributed with the Shapiro-Wilk-Normality test. As the data was normally distributed, we used the two-way ANOVA with post-hoc Bonferroni test to analyze the level of significance $\left({ }^{*} p \leq 0.05,{ }^{* *} p \leq 0.01,{ }^{* * *} p \leq 0.001\right)$.

\section{RESULTS}

\section{Successful Deletion of LRP1 in Hippocampal Astrocytes}

We have previously shown that the deletion of $\operatorname{Lrp} 1$ in neural stem and precursor cells leads to an increased number in GFAPpositive astrocytes (Safina et al., 2016) and that the absence of Lrp1 in astrocytes caused a delayed maturation and an altered neuronal transmission in neurons in vitro (Romeo et al., 2021). Here we were interested how the deletion of astroglial Lrp1 affects the differentiation of astrocytes and the functionality in vivo. Therefore, we used the already described triple transgenic mouse line GLAST::CreERT2xLRP1 (Romeo et al., 2021). In this mouse model LRP1 was specifically deleted in GLAST-positive astrocytes and the recombination events were visualized with the expression of the reporter tdTomato. In this study, we used LRP1deficient animals (GLAST ${ }^{\mathrm{CreERT}} /{ }^{\mathrm{wt}} \operatorname{Rosa} 26^{\mathrm{fl} / \mathrm{fl}} \mathrm{LRP}^{\mathrm{fl} / \mathrm{fl}}$; further referred to as $\mathrm{LRP} 1^{\mathrm{fl} / \mathrm{fl}}$ or knockout) and control animals, with expression of tdTomato (GLAST ${ }^{\text {CreERT2 } / w t}{ }^{\mathrm{wt}} \mathrm{Ra} 26^{\mathrm{fl} / \mathrm{fl}} \mathrm{LRP} 1^{\mathrm{wt}} / \mathrm{wt}$; further referred to as LRP1 $1^{\mathrm{wt} / \mathrm{wt}}$ or control). To induce the conditional knockout, tamoxifen (TAM) was applied to lactating mothers. The application of TAM caused release of CreERT2 from HSP90 and translocation to the nucleus, subsequently followed by the excision of loxP-flanked genes, ( $\operatorname{Lrp} 1$ and the stop codon upstream of Tdtomato to induce the expression of the reporter) (Weber et al., 2001; Mori and Zhang, 2006). First, we evaluated the recombination efficiency in control and conditional knockout mice at three developmental stages via immunohistochemistry (P21, P28 and adult) within the hippocampus. Therefore, we quantified all tdTomato-positive cells divided by the total number of Hoechst-positive cell nuclei in the molecular layers of the hippocampus. The quantification showed that at P21 $92.51 \% \pm 1.73 \%$ of the cells in control animals and $94.21 \% \pm 0.36 \%$ of the cells in the conditional knockout were recombined (see Figures 1A,B). At P28 the recombination efficiency was similar to $\mathrm{P} 21(85.48 \% \pm 1.92 \%$ in the control mice and $90.97 \% \pm 2.13 \%$ in the knockout mice, respectively). The number of recombined cells was stable over time. At the adult stage around $96.77 \% \pm 0.24 \%$ of the cells were still tdTomato-positive in LRP1-deficient animals in comparison to $95.4 \% \pm 0.35 \%$ in control mice. Next, we investigated the number of LRP1- and tdTomato-double positive cells in both conditions to evaluate the knockout efficiency. Therefore, the number of LRP1- and tdTomato-double positive cells were divided by the number of all tdTomato-positive cells. The expression of LRP1 was significantly decreased in the knockout condition compared to the control condition (P21: $\mathrm{LRP}^{\mathrm{wt} / \mathrm{wt}} 59.76 \% \pm 2.48 \%, \mathrm{LRP}^{\mathrm{fl} / \mathrm{fl}} 5.83 \% \pm 1.36 \%, p \leq 0.0001$; P28: $\mathrm{LRP}^{\mathrm{wt} / \mathrm{wt}} 71.05 \% \pm 3.8 \%, \mathrm{LRP}^{\mathrm{fl} / \mathrm{fl}} 7.15 \% \pm 1.88 \%$, $p \leq 0.0001$; adult: $\mathrm{LRP}^{\mathrm{wt} / \mathrm{wt}} 63.45 \% \pm 3.82 \%, \mathrm{LRP}^{\mathrm{fl} / \mathrm{fl}}$ $2.51 \% \pm 0.56 \%, p \leq 0.0001$ ) at all investigated time points (see Figures 1A,C). To further validate the downregulation of LRP1 in the knockout animals, we performed PCR and western blot analysis. The relative expression of LRP1 was not affected by the knockout induction (see Figures 1D,E). Furthermore, the western blot analysis showed no significant alterations in the protein expression of LRP1 (see Figures 1F,G). However, it needs to be considered that hippocampal tissue was used including other cell types expressing LRP1.

To confirm that the deletion of LRP1 only affected the astroglial lineage an immunohistochemical staining against NeuN and CC1 was performed to exclude neurons and oligodendrocytes. Therefore, knockout tissue of P28 mice was used and almost no co-localization of tdTomato with either NeuN-positive neurons or CC1-positive oligodendrocytes were observed, meaning that the vast majority of cells lacking LRP1 were astrocytes (see Figure 2A). Additionally, we analyzed the weight of LRP1-deficient animals and compared the values to control animals and we saw no difference (see Figure 2B). Due to the similar structure and comparable functions in regard to endocytosis (Auderset et al., 2016), we were interested if the LDL-family member LRP2 was upregulated in hippocampal tissue of LRP1-deficient animals during the development as a compensatory effect. We investigated the expression of the LDLfamily member via PCR. The expression of LRP2 was not altered in LRP1-deficient hippocampal tissue in comparison to control tissue (see Figures 2C,D).

\section{LRP1 Did Not Influence Proliferation of Hippocampal Astrocytes at P21}

Due to the high number of potential ligands LRP1 is known to influence several cellular processes including proliferation. Therefore, we investigated the proliferation capacity of recombined astrocytes via immunohistochemistry (see Figure 3A). The numbers of phospho-Histone $\mathrm{H} 3$ (PH3)- and tdTomato-double positive cells were quantified (see Figure 3B). The quantification revealed that the absence of LRP1 caused no altered proliferation rate. Therefore, the number of PH3- and tdTomato-positive cells were comparable in both conditions.

\section{No Changes in Astroglial Maturation After Loss of LRP1}

We analyzed whether the deletion of LRP1 had any influence on the gene expression of several markers specific for the late precursor or immature astroglial stage (see Figure 4). To identify late precursor cells favoring an astroglial fate, we used Glast 


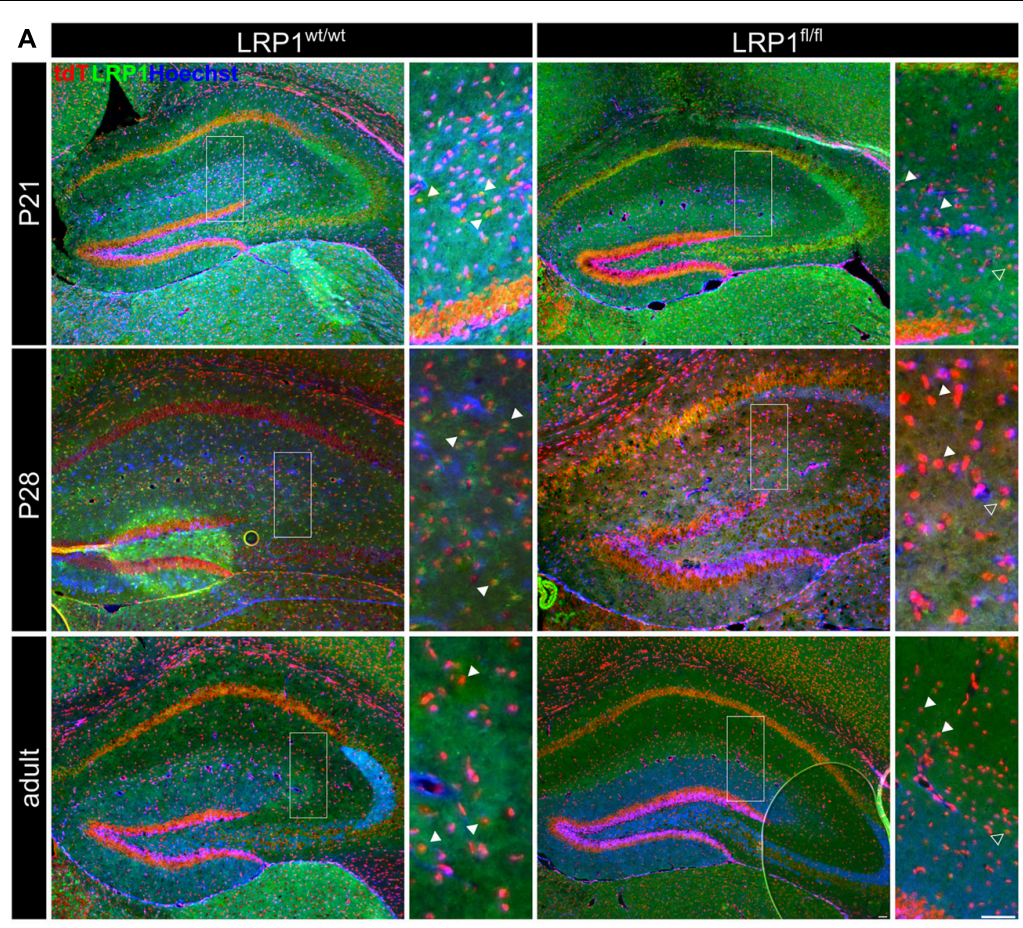

B

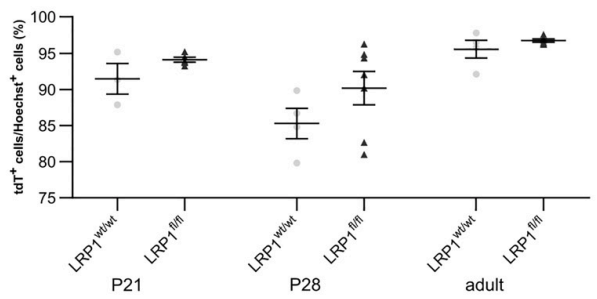

D

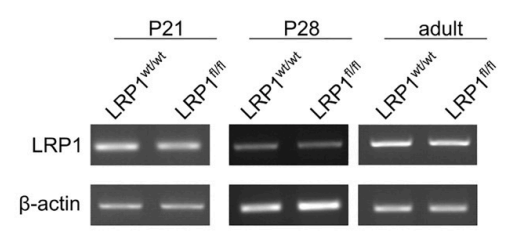

$\mathbf{F}$

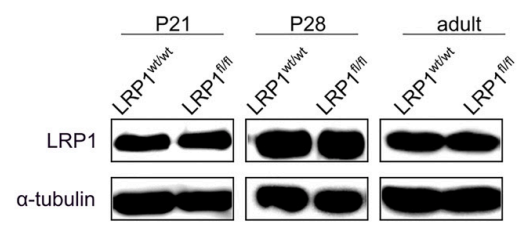

C

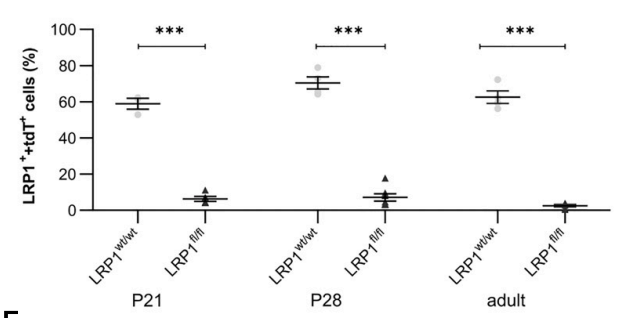

E

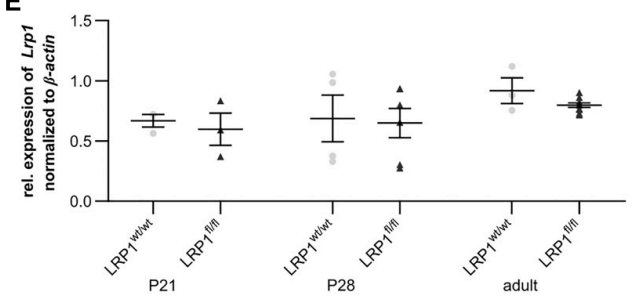

G

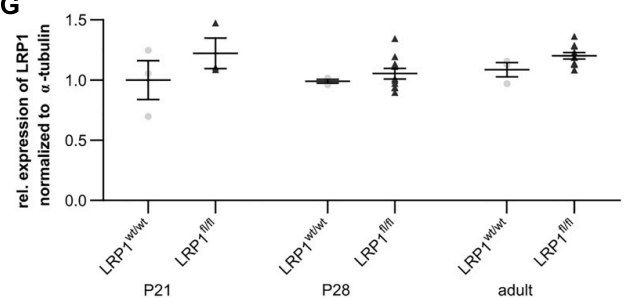

FIGURE 1 | Knockout induction via lactating mothers was successful in vivo. The immunohistochemical staining against LRP1 (green) and tdTomato (red) revealed the expression of LRP1 by recombined astrocytes during development indicated by the arrowhead (A). The recombination rate differed between 85 and $97 \%$ during development in LRP1-deficient and control hippocampi (B). The knockout efficiency was evaluated by the quantification of LRP1-and tdTomato-double positive cells divided by the total number of recombined cells within the hippocampus. Here the quantification showed that the number of LRP1-expressing recombined cells was significantly decreased in the knockout compared to the control (C). Furthermore, the knockout induction was evaluated via RT-PCR (D). The gene expression of Lrp1 was not altered in LRP1-deficient hippocampi during development compared to control tissue (E). As a last approach the protein expression of LRP1 was analyzed with western blot analysis (F). The protein expression was comparable in both conditions during development (G) (Scale bar is $50 \mu \mathrm{m}$; mean \pm SEM; two-way ANOVA with post-hoc Bonferroni test; ${ }^{*} p<0.05,{ }^{\star \star} p<0.01,{ }^{\star \star \star} p<0.001$; for $N$-values see Table 2). 
TABLE 2 | Number of biological N for each performed experiment.

\begin{tabular}{|c|c|c|}
\hline & LRP1wt/wt & $\operatorname{LRP}^{\mathrm{fl} / \mathrm{fl}}$ \\
\hline Immunohistochemistry & $\begin{array}{l}\text { P21: } N=3 \\
\text { P28: } N=4 \\
\text { Adult: } N=4\end{array}$ & $\begin{array}{c}\text { P21: } N=5 \\
\text { P28: } N=7 \\
\text { Adult: } N=6\end{array}$ \\
\hline PCR/western blot & $\begin{array}{l}\text { P21: } N=3 \\
\text { P28: } N=4 \\
\text { Adult: } N=3\end{array}$ & $\begin{aligned} \text { P21: } N & =3 \\
\text { P28: } N & =6 \\
\text { Adult: } N & =10\end{aligned}$ \\
\hline Weight & $\begin{array}{l}\text { P21: } N=6 \\
\text { P28: } N=3 \\
\text { Adult: } N=9\end{array}$ & $\begin{array}{c}\text { P21: } N=8 \\
\text { P28: } N=17 \\
\text { Adult: } N=17\end{array}$ \\
\hline Behavioral tests & $N=5$ & $N=6$ \\
\hline
\end{tabular}

(see Figures 4A,B) and Fgfr-3 (see Figures 4A,C), whereas immature astrocytes were investigated via the expression of $A q p$ 4 (see Figures 4A,D) as well as Aldh1l1 (see Figures 4A,E). The gene expression analysis revealed no changes of any marker in hippocampal tissue of LRP1-deficient animals compared to the control condition, highlighting that LRP1 has no influence on astroglial maturation based on the analyzed markers.

\section{Decreased Quantity of GFAP-Positive Astrocytes in the Hippocampus at P21}

We previously reported that the deletion of $L r p 1$ in neural stem and progenitor cells (NSPCs) caused an increased number of glial fibrillary acidic protein (GFAP)-positive astrocytes (Safina et al., 2016; Bres et al., 2020). However, LRP1-deficient astrocytes did not show a reactive phenotype in vitro (Romeo et al., 2021). Via the immunohistochemical staining against GFAP, we wanted to investigate the influence of the LRP1 deletion in regard to a reactive phenotype and in terms of maturation, where GFAP is mainly expressed by mature astrocytes in vivo (see Figure 5A). The quantification of GFAP- and tdTomato-double positive cells revealed that the deletion of LRP1 led to a significantly decreased number of double positive cells $(19.73 \% \pm 1.68 \%)$ in comparison to the control tissue $(39.11 \% \pm 2.24 \% ; p \leq 0.01)$ at $\mathrm{P} 21$ (see Figure 5B). With ongoing development, the number of GFAPand tdTomato-positive astrocytes increased in the knockout mice and was comparable to the control condition reaching adulthood where the numbers stabilized.

Furthermore, mRNA expression of Gfap was investigated to support the findings of the immunohistochemical staining (see Figure 5C). Contrary to immunohistochemical data, the mRNA analysis showed that the astroglial loss of Lrp1 had no effect on Gfap mRNA expression at any developmental stage in the hippocampus compared to the control tissue (see Figure 5D).

Additionally, the number of $\mathrm{S} 100$ calcium binding protein $\beta$ (S100)-positive cells was evaluated to investigate the maturation of LRP1-depleted astrocytes (see Figure 6A). The immunohistochemical staining showed that the number of S100and tdTomato-positive cells was significantly downregulated in LRP1-deficient hippocampi $(16.55 \% \pm 2.88 \%)$ when compared to control tissue $(28.45 \% \pm 2.44 \% ; p \leq 0.05)$ at P21 (see Figure 6B). The number of double positive cells was comparable in control and LRP1-deficient mice with ongoing development. However, similar to GFAP, the mRNA expression of $S 100$ was not affected by the deletion of LRP1 in astrocytes (see Figures 6C,D).

The data indicates that LRP1 mainly influences the maturation of astrocytes in early development in the hippocampus. However, these differences were compensated with increasing age of the animals. Additionally, the regulation by the knockout induction occurred mainly on protein level as the mRNA expression was not affected by the absence of LRP1, contrary to the number of marker-positive cells.

\section{Expression of Glutamate Transporter GLT-1 Was Not Altered in Astrocytes Upon LRP1 Deletion}

Astrocytes have diverse functions and can contribute to different cellular processes. They are involved in synaptic transmission via the uptake of neurotransmitters, like glutamate, out of the synaptic cleft. Therefore, the expression of the glutamate transporter 1 (GLT-1) on the cell surface of LRP1-deficient astrocytes was investigated (see Figure 7A). The quantification of the immunohistochemical staining revealed no alterations in the number of GLT-1- and tdTomato-positive cells in early development at P21 (see Figure 7B). With increasing age, GLT-1 becomes the main glutamate transporter and the number of GLT1-positive astrocytes should increase. Our analysis showed that the number of GLT-1- and tdTomato-positive cells increased in controls and LRP1-deficient mice but slightly decreased reaching adulthood. Although, no changes in Glt-1 mRNA expression were observed (see Figure 7C). The analysis showed no alterations in the expression of Glt-1 in LRP1-deficient hippocampal tissue at all three investigated timepoints in comparison to the control tissue (see Figure 7D). Furthermore, the protein expression of GLT-1 was not affected by the depletion of LRP1 as well (see Supplementary Figures S2A,B).

\section{LRP1-Deficient Hippocampal Tissue Showed Significantly Decreased Activity of the Ras/Raf/MEK/Erk-Signaling Pathway at P21}

The deletion of LRP1 in astrocytes mainly influenced the maturation at an early developmental timepoint. As previously described the maturation of glial cells is dependent on the Ras/Raf/MEK/Erk and PI3K/Akt signaling cascade. Therefore, we were interested whether the phosphorylation of the signaling molecules Erk1/2 and Akt were influenced by the deletion of LRP1 in astrocytes and therefore might explain the delayed maturation we observed at P21 (see Figure 8). The western blot analysis showed a significant downregulation of the active phospho-Erk (pErk) in P21 hippocampal tissue after astroglial loss of LRP1 (0.418 \pm 0.197$)$ compared to controls (1.138 \pm 0092 ; $p \leq 0.05$ ) (see Figures 8A,B). However, with ongoing maturation the protein expression was comparable in both conditions. The activation of pErk in astrocytes was strongly decreased as we investigated hippocampal tissue including other cell types, also expressing several signaling molecules, such as pErk. 

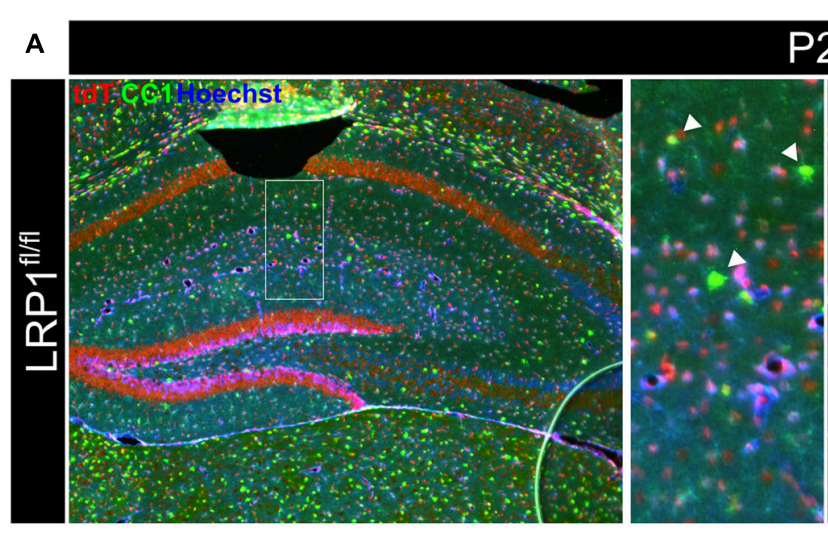

\section{P28}

B

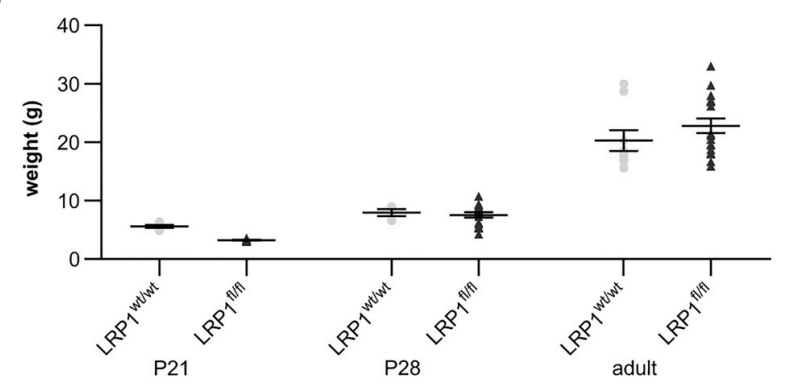

C

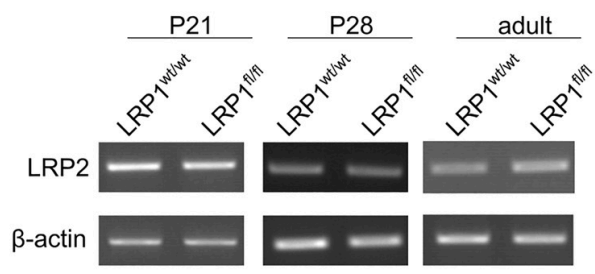

D

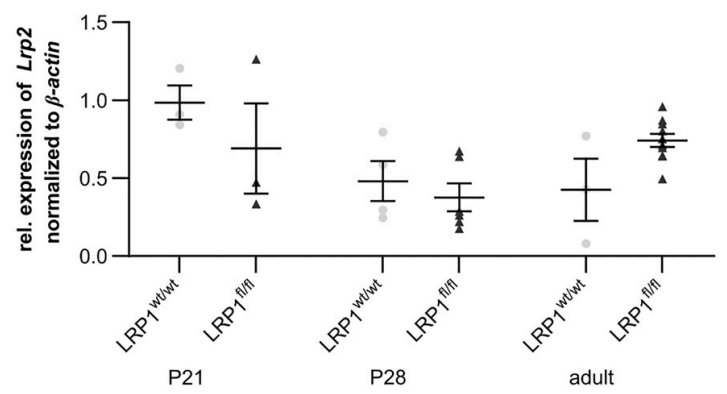

FIGURE 2 | Characterization of the knockout in vivo. To confirm that the deletion of LRP1 was restricted to the astrocytic lineage, an immunohistochemical staining against neurons (NeuN; green) and oligodendrocytes (CC1; green) was performed (A). There was no colocalization of both cell-specific markers with the expression of tdTomato in recombined cells indicated by the arrowhead leading to the assumption that the deletion of LRP1 only occurred in astrocytes. To further characterize the newly generated mouse line, the weights of the TAM-receiving animals were compared (B). The statistical analysis revealed no changes in the weight of knockout animals compared to the control. As the deletion of one member of the LDL-family might results in an increased expression of other members, the expression of Lrp2 (C,D) was investigated. However, the knockout induction of LRP1 caused no altered expression of LRP2 (Scale bar is $50 \mu \mathrm{m}$; mean \pm SEM; two-way ANOVA with post-hoc Bonferroni test; for $N$-values see Table 2).

The expression of pAkt was not influenced by the deletion of LRP1 in astrocytes in comparison to the controls at any investigated timepoint (see Figures 8C,D) as we could find no alterations in the gene expression of $A k t$ and Mtor after loss of LRP1 (data not shown).

\section{Deletion of Astrocytic LRP1 Negatively Influenced Activity of Hippocampal Neurons in vivo}

To further investigate the effect of LRP1 loss in astrocytes to hippocampal tissue, we investigated the activity of neurons via the staining against c-Fos, marking activated neurons (see
Figure 9A). Thereby, we deduced whether the metabolic supply of neurons via astrocytes was influenced by the deletion of Lrp1. All c-Fos-positive cells were quantified and the number of positive cells per $\mathrm{mm}^{2}$ was measured (see Figure 9B). The quantification showed a significantly decreased number of c-Fospositive neurons after loss of LRP1 (10.53 cells $/ \mathrm{mm}^{2} \pm 3.15$ cells $/ \mathrm{mm}^{2}$ ) when compared to the control condition (41.90 cells $/ \mathrm{mm}^{2} \pm 3.41$ cells $/ \mathrm{mm}^{2} ; p \leq 0.01$ ) at P21. With ongoing maturation, the number of c-Fos-positive neurons were similar in both conditions.

Furthermore, the expression of subunits of the glutamate receptors $\alpha$-amino-3-hydroxy-5-methyl-4-isoxazolepropionic acid (AMPA) and N-methyl-D-aspartate (NMDA) were 


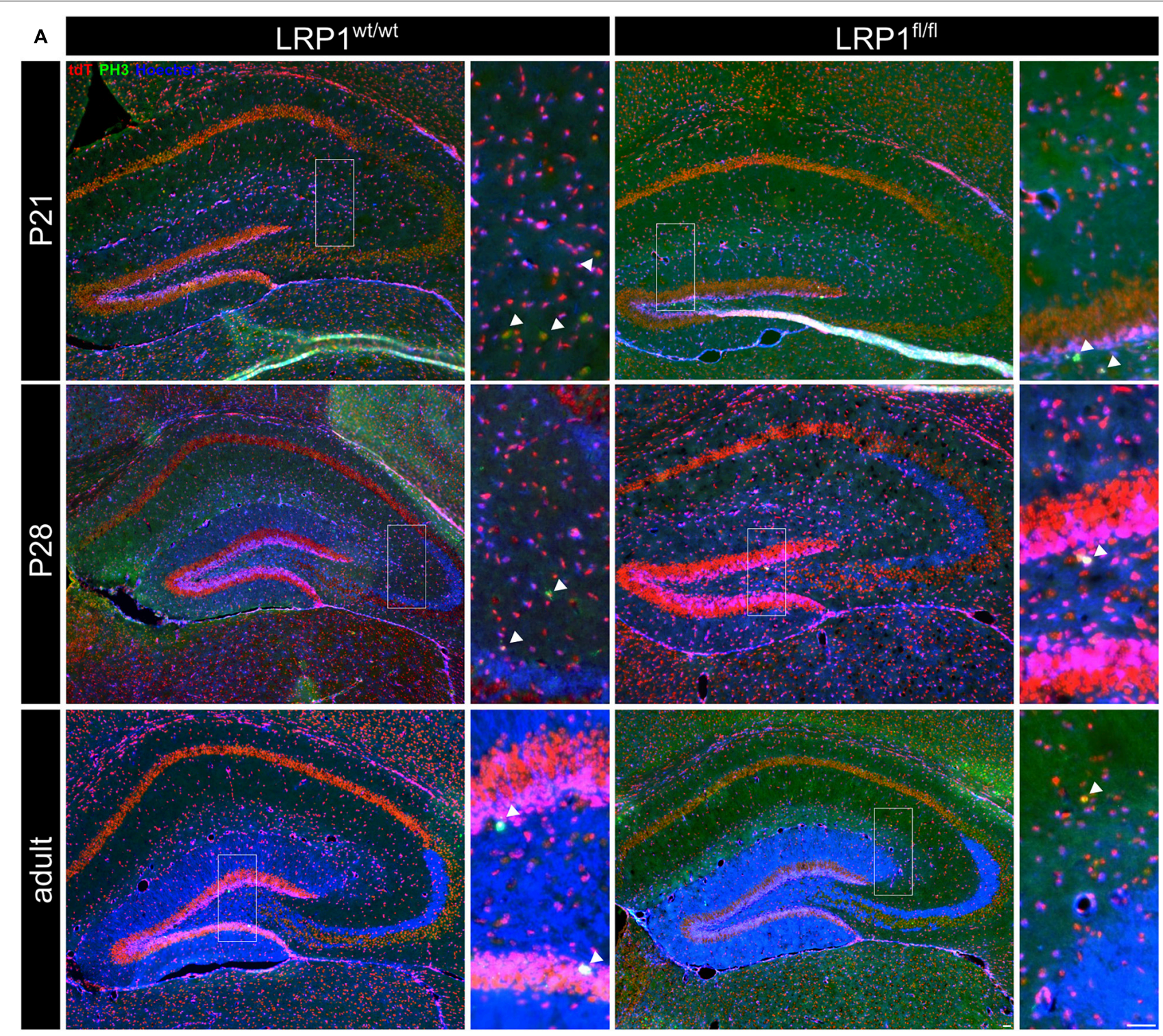

B

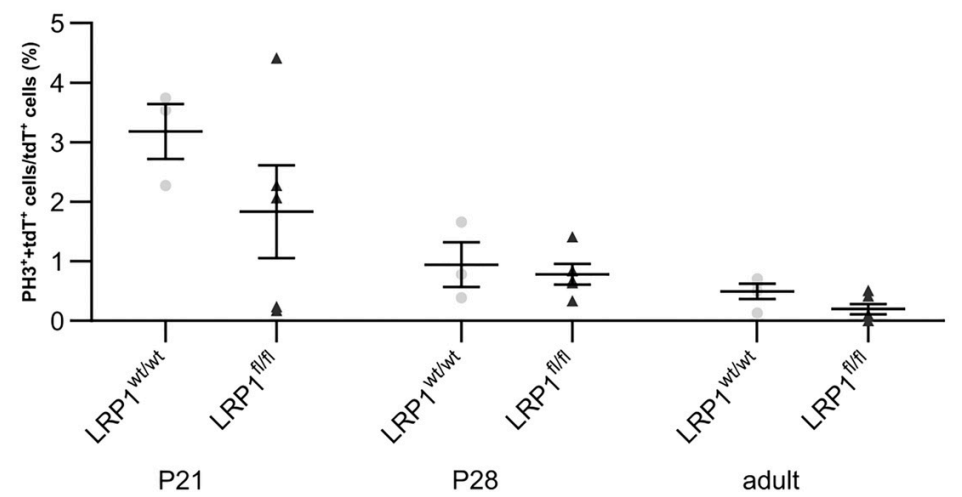

FIGURE 3 | Proliferation capacity of LRP1-deficient astrocytes was not affected. The proliferation rate was evaluated with an immunohistochemical staining against phospho-Histone H3 ( $\mathrm{PH} 3$; green) and tdTomato (A, red). Double-positive cells indicated by the arrowhead were quantified and the analysis showed that the numbers of proliferation events were comparable in both conditions (B. Scale bar is $50 \mu \mathrm{m}$; mean \pm SEM; two-way ANOVA with post-hoc Bonferroni test; for $N$-values see Table 2). 


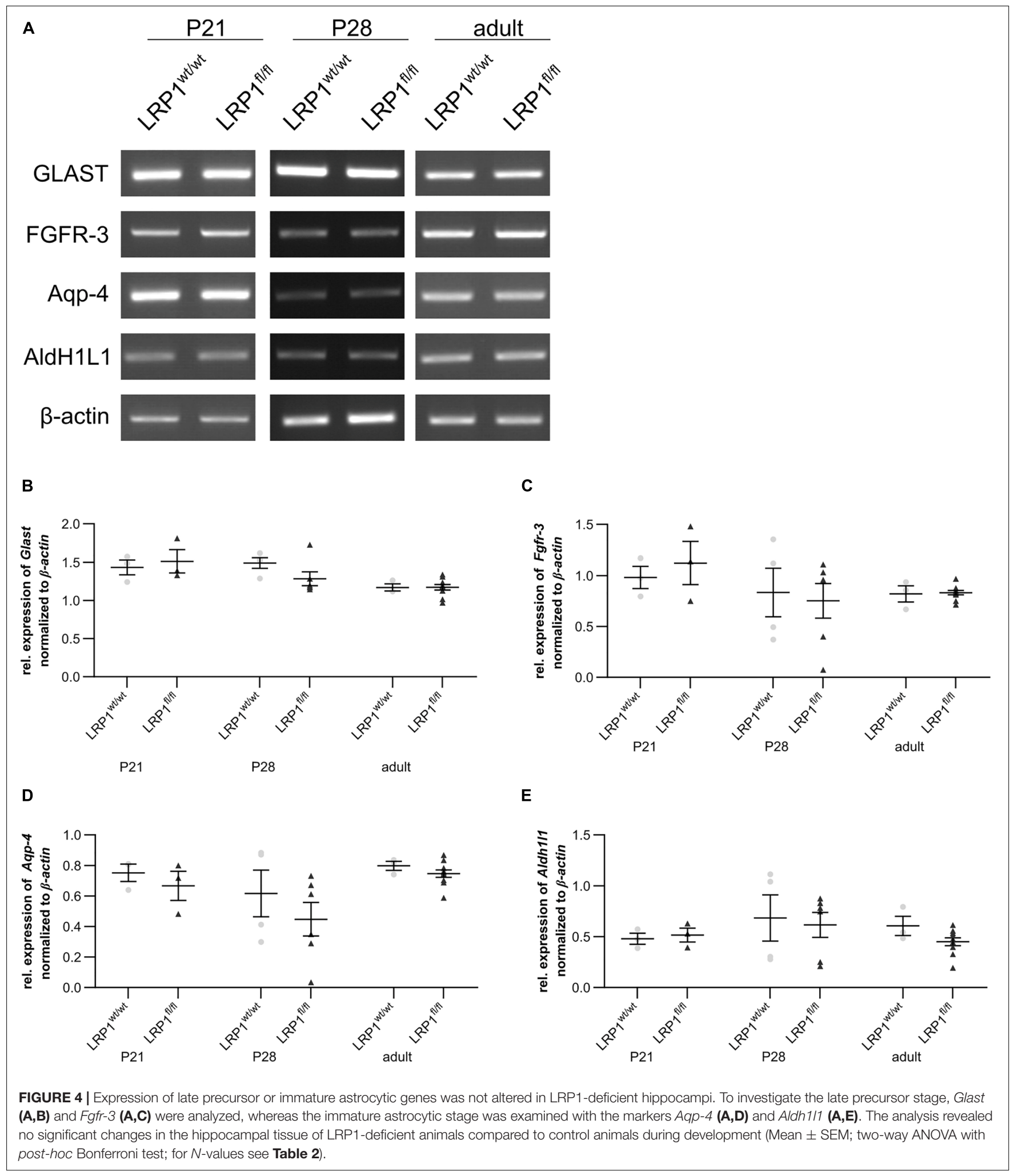

investigated via RT-PCR (Figure 9C). We investigated the gene expression of the AMPAR subunits Gria1 and Gria2 (see Figures 9D,E) as well as the NMDAR subunits Grin1, Grin2a, and Grin $2 b$ (see Figures $\mathbf{9} \mathbf{F}-\mathbf{H}$ ) as it was previously proposed that these subunits can be influenced via LRP1. However, the expression analysis revealed no alterations in the gene expression of the receptor subunits in LRP1-deficient hippocampal tissue compared to the control during development. 


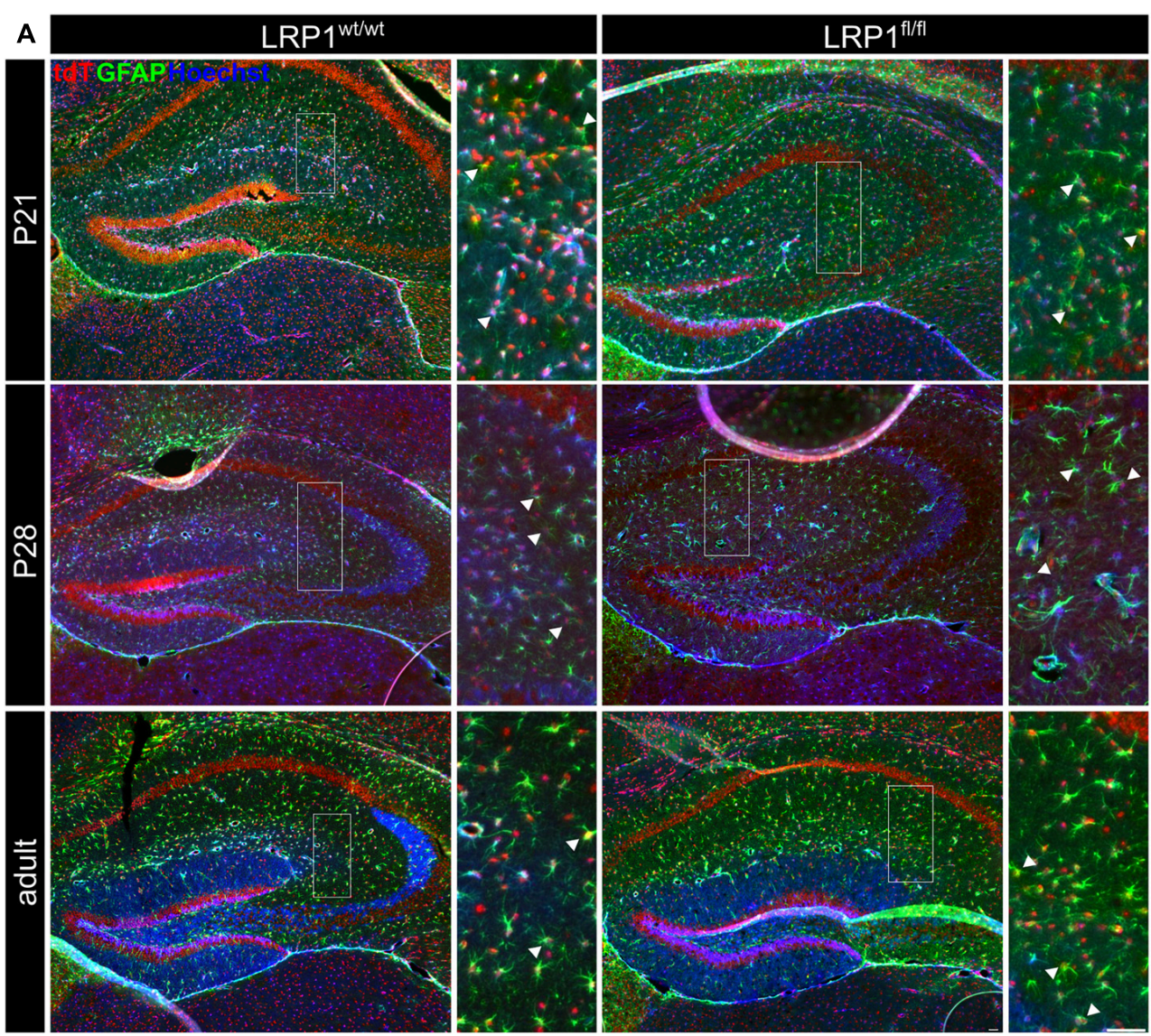

B

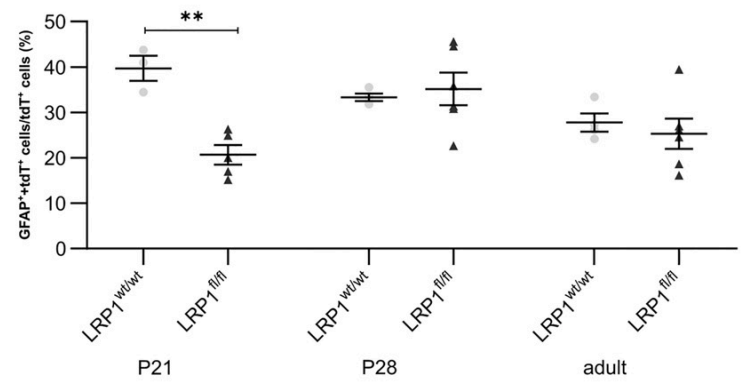

C

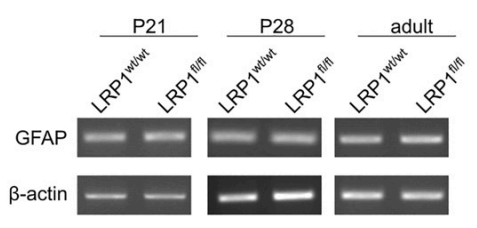

D

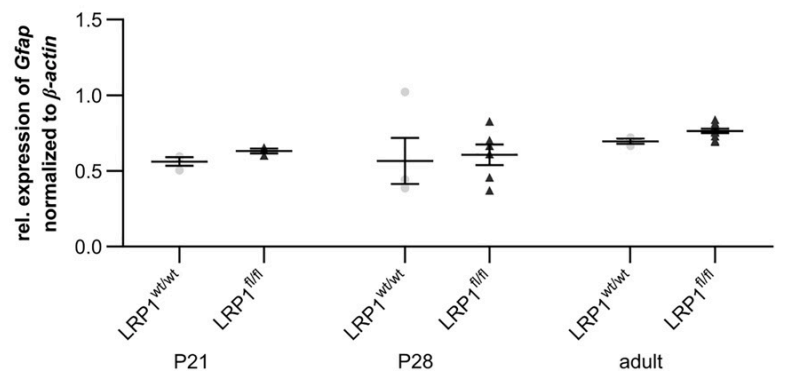

FIGURE 5 | LRP1 depletion in astrocytes resulted in no reactive phenotype. The immunohistochemical staining against GFAP (green) and tdTomato (red) revealed no reactive morphology (A). The quantification of GFAP- and tdTomato-positive cells indicated by the arrowhead showed no changes in the number of double-positive cells in the hippocampi upon LRP1 deletion during development (B). Additionally, RT-PCR analysis was performed to investigate whether the Gfap gene expression was altered (C). However, the statistical analysis showed no differences between the knockout and control condition at all three investigated timepoints (D) (Scale bar is $50 \mu \mathrm{m}$; mean \pm SEM; two-way ANOVA with post-hoc Bonferroni test; for $N$-values see Table 2). 

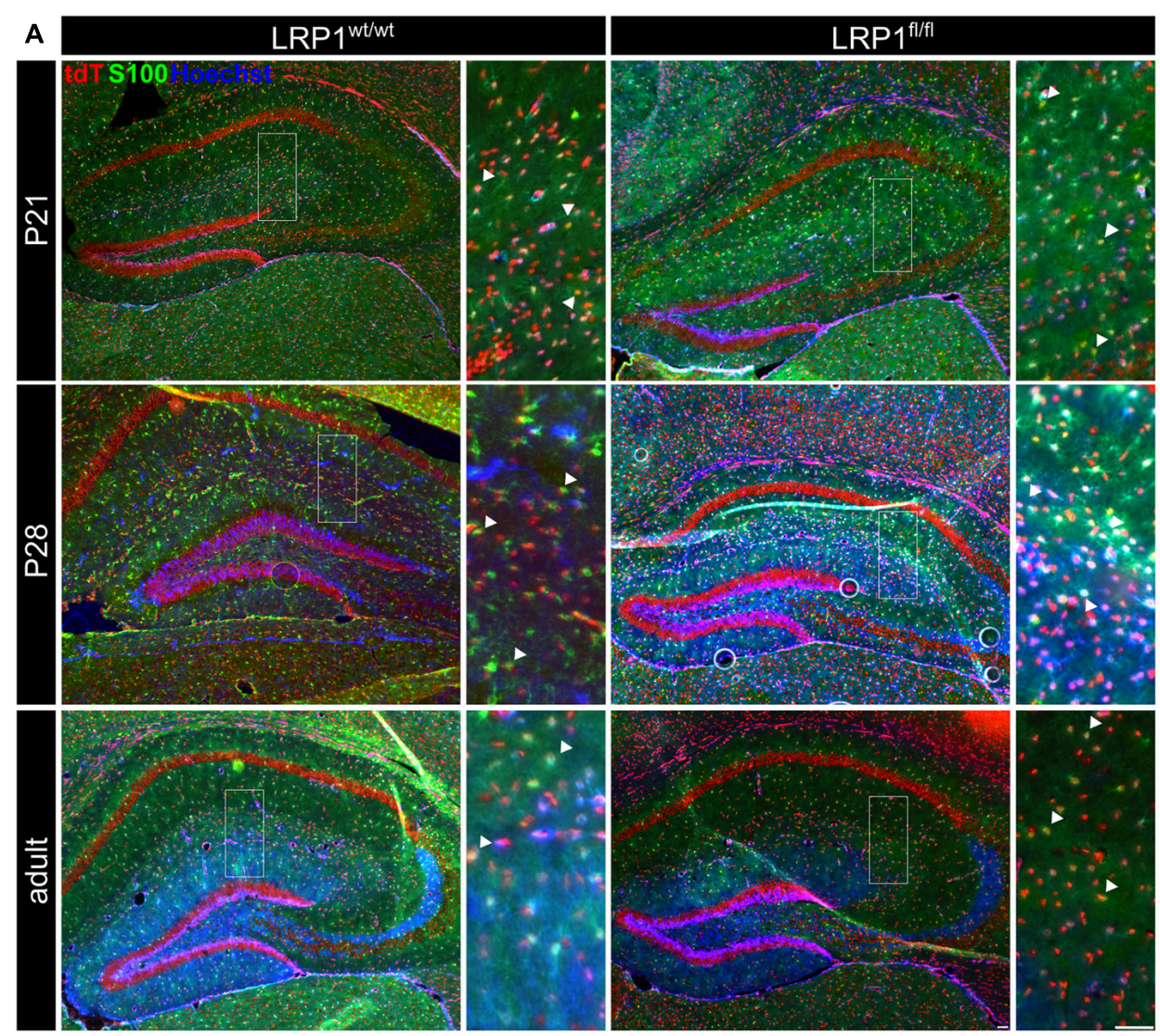

B

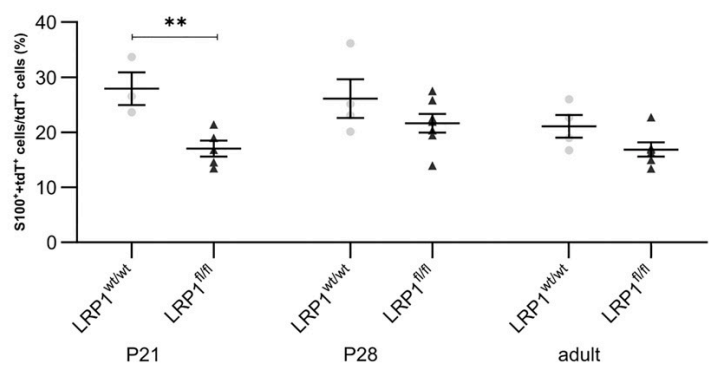

C

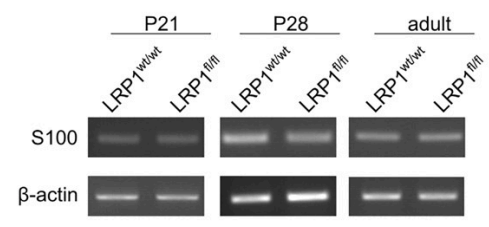

\section{D}

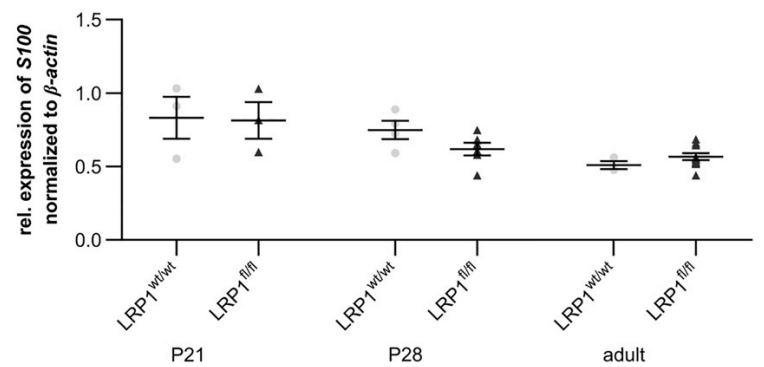

FIGURE 6 | Maturation of LRP1-deficient astrocytes was affected at the beginning of development. To further investigate the maturation of astrocytes upon LRP1 deletion, an immunohistochemical staining against S100 (green) and tdTomato was performed (A). The quantification showed a significantly decreased number of double-positive cells indicated by the arrowhead within the hippocampi of LRP1-deficient animals at P21 compared to the control condition (B). However, with ongoing development the numbers were comparable in both conditions. To support the findings of the staining, RT-PCR analysis was performed to analyze S100 gene expression (C). Nevertheless, the expression was not altered upon LRP1 deletion in hippocampal tissue at all three investigated timepoints (D) (Scale bar is 50 $\mu \mathrm{m}$; mean \pm SEM; two-way ANOVA with post-hoc Bonferroni test; for $N$-values see Table 2). 


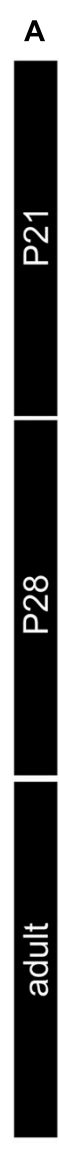

A LRP1wt/wt

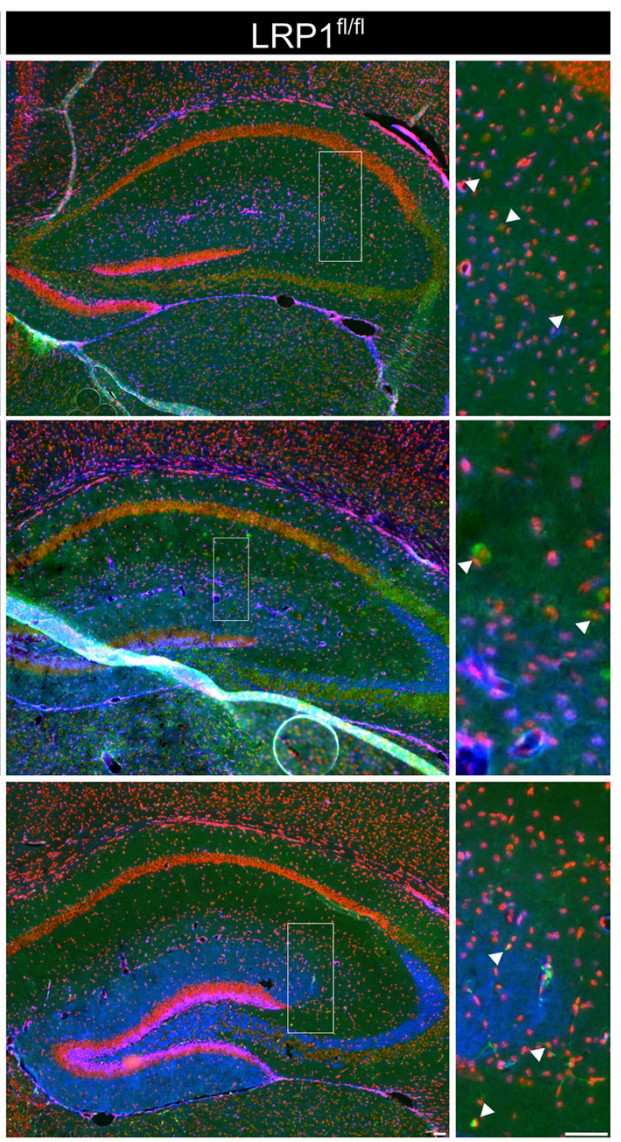

B

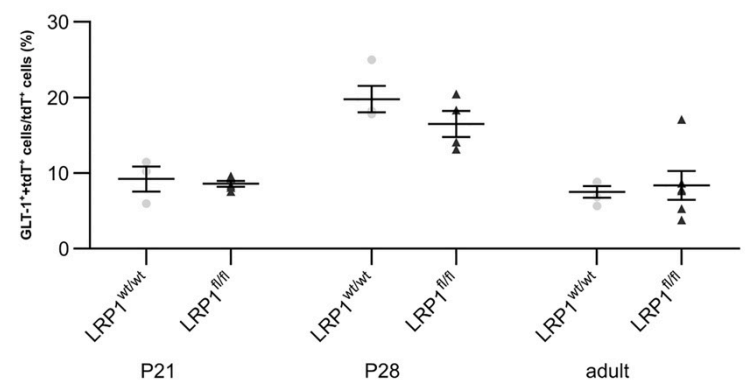

C

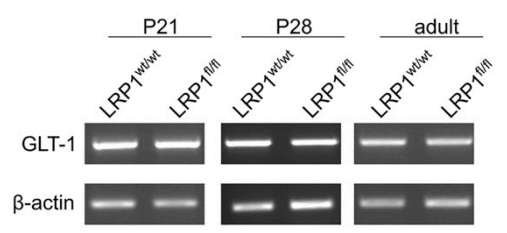

D

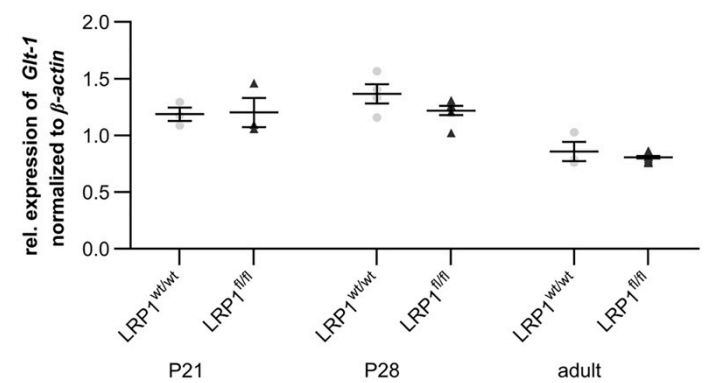

FIGURE 7 | Glutamate transporter expression was not influenced by the deletion of LRP1. As a next approach, the number of GLT-1 (green) and tdTomato-expressing (red) cells was analyzed (A). The statistical evaluation showed no alterations in the number of double-positive cells indicated by the arrowhead in LRP1-depleted tissue compared to the control condition during development (B). Also, the gene expression of G/t-1 was not affected by the deletion of LRP1 in hippocampal tissue (C,D) (Scale bar is $50 \mu \mathrm{m}$; mean \pm SEM; two-way ANOVA with post-hoc Bonferroni test; for $N$-values see Table 2). 


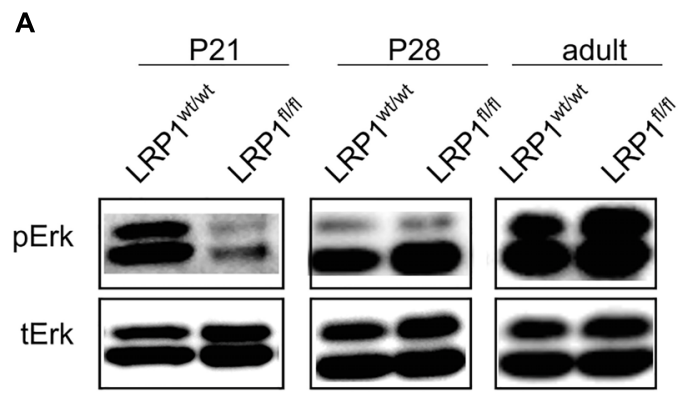

C

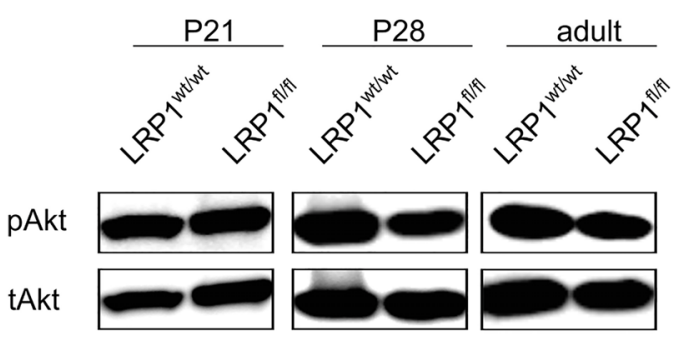

B

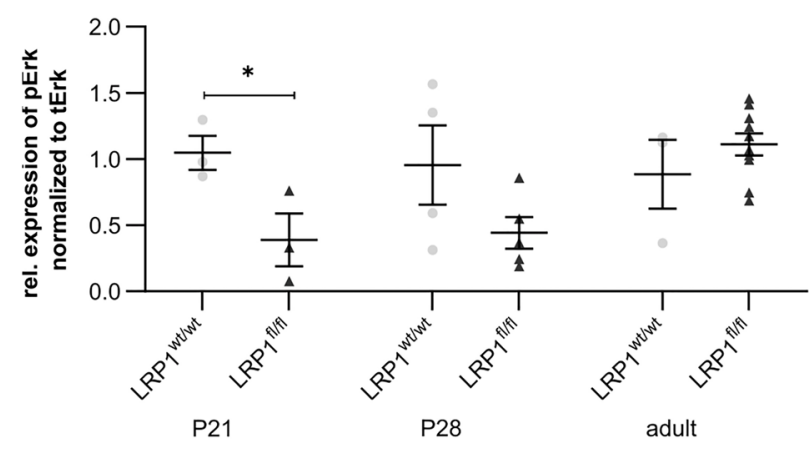

D

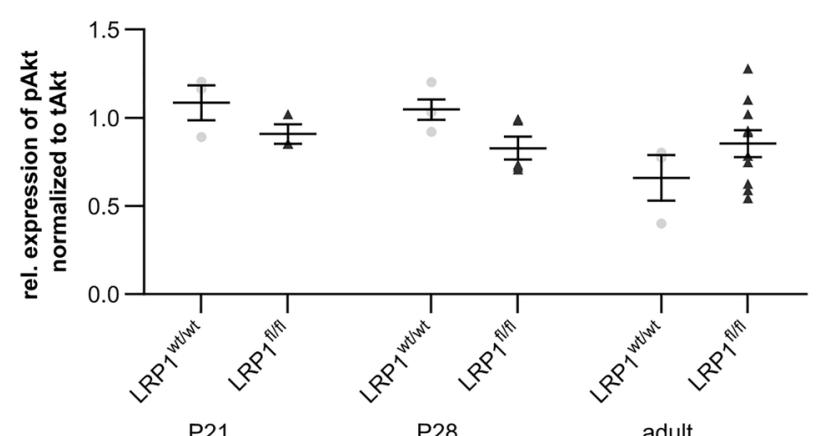

FIGURE 8 | Astrocytic LRP1 influences the activation of the signaling pathway Ras/Raf/MEK/Erk in hippocampal tissue. The phosphorylation of the signaling molecules Erk1/2 and Akt were investigated via western blot analysis. Here, hippocampal tissue was used and the knockout condition showed a significantly decreased protein expression of pErk normalized to tErk at P21 (A,B). However, with ongoing development the activation was comparable between both conditions. The deletion of LRP1 in astrocytes had no influence on the phosphorylation of the signaling molecule Akt (C,D) (Mean \pm SEM; two-way ANOVA with post-hoc Bonferroni test; for $N$-values see Table 2).

\section{Deletion of Astrocytic LRP1 Had No Influence on Motor Coordination or Cognitive Abilities in Adult Animals}

To assess if the astrocytic deletion of LRP1 had any influence of the behavior or the motor coordination on adult knockout animals several tests were performed. Fine motor coordination and balance were investigated with the beam walk test. The first investigated parameter was the time that was needed by the animals to cross the beam (see Figure 10A), the second parameter was the time left immobile until they started walking on the beam for the first time (see Figure 10B) and the last parameter were the slips of the hindlimbs, either right or left, of the animals while crossing the beam (see Figure 10C). In general, the LRP1-deficient animals exhibited no motor dysfunctions or imbalance in comparison to the control animals. However, the number of slips of the hindlimbs were slightly decreased compared to the control animals, hinting an increased balance. Next, we used the hangwire test to evaluate strength with little coordination. The knockout animals performed in the same way as the control animals in this task (see Figure 10D). Additionally, the motor coordination and balance were analyzed with the pole test. The time needed to descend down to the cage was not altered in the knockout group compared to control animals (see Figure 10E). The last motor coordination test was the rotarod.
The investigated parameters here were the time when the animals fell down (see Figure 10F) as well as the speed of the rod when the animals fell down (see Figure 10G). Again, we saw no differences in the motor coordination of LRP1-depleted animals.

The cognitive abilities of the LRP1-deficient animals were assessed with the T-maze test (see Figure $\mathbf{1 0 H}$ ) and the novel object recognition (NOR) test (see Figure 10I). These two tests revealed that the knockout animals showed no cognitive disabilities in comparison to the control group.

These findings lead to the assumption that the astrocytic deletion of LRP1 had no effect on the motor coordination as well as on the cognitive abilities of adult animals.

\section{DISCUSSION}

We used the newly generated mouse model with an inducible loss of astroglial LRP1 to examine how the maturation and maintenance of astrocytes is influenced by astroglial LRP1. Therefore, we decided to investigate the maturation on protein (immunohistochemistry, western blot) and mRNA level (RT-PCR). The application of Tamoxifen caused a significantly decreased number of LRP1-expressing cells in the knockout condition compared to the control condition. However, western blot analysis revealed no significant downregulation. 

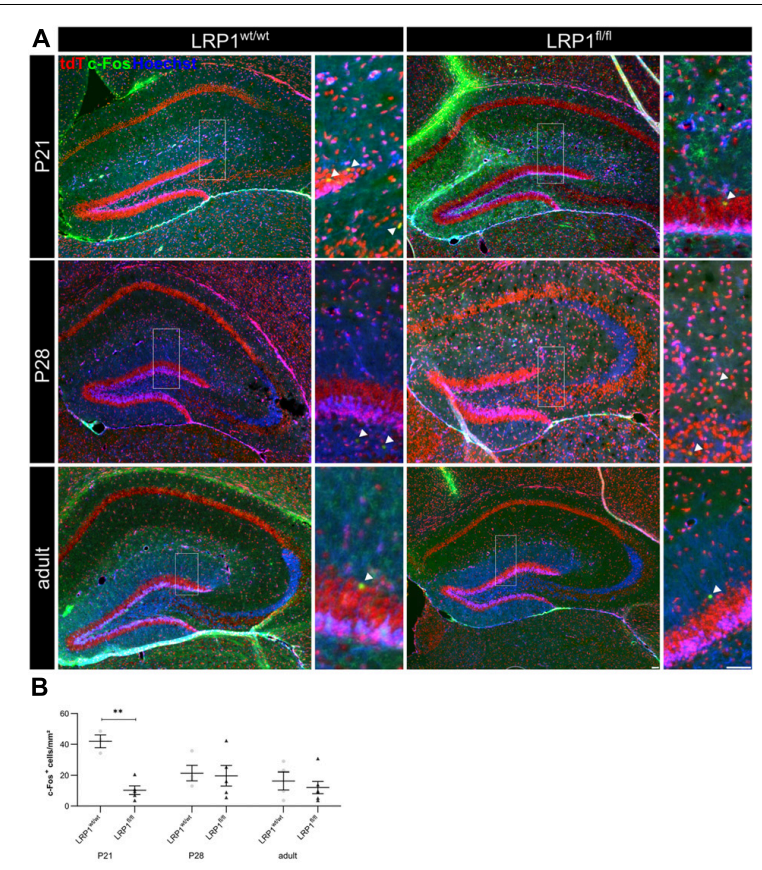

C

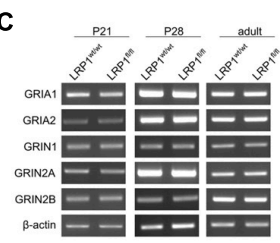

E
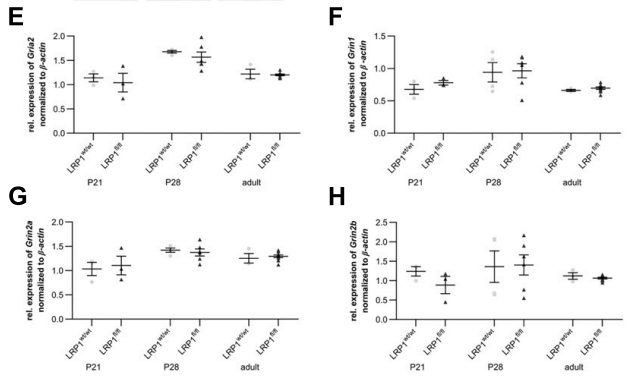

FIGURE 9 | Neuronal activity was negatively influenced in the knockout condition. To assess the neuronal activity within the hippocampus of LRP1-deficient animals, an immunohistochemical staining against c-Fos (green) and tdTomato (red) was performed (A). The quantification of all c-Fos-positive cells indicated by the arrowhead within the hippocampus divided by the area of the hippocampus revealed a significantly decreased number of activated neurons in the knockout condition at P21 compared to the control (B). However, with ongoing maturation the differences were compensated. To further validate the effect of astrocytic LRP1 on the neuronal activity RT-PCR was performed (C). The expression of AMPAR subunits Gria1 (D) and Gria2 (E) was not altered in hippocampal knockout tissue.

Furthermore, the NMDAR subunits Grin1 (F), Grin2a (G) and Grin2b (H) also showed no altered expression profile upon the deletion of LRP1 during development (Scale bar is $50 \mu \mathrm{m}$; mean \pm SEM; two-way ANOVA with post-hoc Bonferroni test; ${ }^{\star} p<0.05$, ${ }^{\star \star} p<0.01$, ${ }^{\star * *} p<0.001$; for $N$-values see Table 2).

Nevertheless, the analysis was performed with hippocampal tissue, including other cell types, such as neurons and microglia, beside the knockout-affected astrocytes. Previous studies showed that $50 \%$ of LRP1 protein is expressed by neurons (Shinohara et al., 2017; Liu et al., 2019), possibly masking the deletion of LRP1 in astrocytes. The further analysis of the knockout specificity revealed that only negligible amount of either NeuNor CC1-positive cells were expressing tdTomato. However, previous studies reported that CC1 was detectable in a small proportion of astrocytes (Bhat et al., 1996; Fuss et al., 2000; Mori et al., 2006; Bin et al., 2016). Mori et al. (2005) have reviewed the concurring expression of astrocyte-specific markers also in radial glia cells. Recombination under the GFAP promotor was detectable in neurons derived from radial glia cells (Malatesta et al., 2003). Leading to the assumption that tdTomato- and NeuN-double positive cells observed in the described animal model might resemble newborn neurons derived from radial glia cells located in the dentate gyrus affected by the recombination. However, as neither neurons nor oligodendrocytes are expressing the used astrocyte-specific markers, the analysis performed in this study resembled the effect of LRP1 on the maturation of astrocytes.

After successful confirmation of astroglia-specific LRP1 loss in vivo, we investigated the proliferation behavior of recombined cells within the hippocampus during development and observed no changes in astroglial proliferation rate. We previously reported that the proliferation of neural stem and precursor cells in vitro was negatively influenced by the deletion of LRP1 (Safina et al., 2016). However, the role of LRP1 in regard to proliferation is opposed, depending on the cell type and tissue.

We tracked the astroglial differentiation with stage-specific markers according to Wiese et al. (2012). However, the gene analysis revealed no significant alterations in regard to the expression of late precursor markers, such as GLAST and FGFR-3, nor to immature astrocytes markers, such as AldH1L1 and Aqp-4. Nevertheless, the protein expression is not always correlating to the mRNA levels, as the relationship between mRNA to protein synthesis is objected to fluctuations caused by different factors, such as resource availability (Liu et al., 2016). Therefore, the number of positive cells might be altered, whereas the mRNA levels were not affected by the LRP1 deletion.

As a next step, we investigated mature astrocytes with immunohistochemistry. Here we used GFAP, a prototypical marker for mature astrocytes and a reliable maker for reactive astrogliosis (Pekny et al., 1995; Pekny and Pekna, 2004; Herrmann et al., 2008). Our analysis showed a significantly decreased number of recombined cells expressing GFAP at P21 in knockout mice compared to controls. Barcelona et al. (2011) showed that the expression of GFAP in retinal Müller glia was induced by application of $\alpha 2$-macroglobulin. This effect was abolished by using the LRP1 inhibitor RAP. Therefore, the binding of $\alpha 2$-macroglobulin to LRP1 facilitates the expression of GFAP. The deletion of LRP1 in astroglia might cause the same effect, leading to a decreased number of GFAP-positive astrocytes observed in our animal model. Furthermore, we assessed mature astrocytes with S100 and again the number of tdTomato- and S100-double positive cells was significantly decreased in the hippocampus at P21. However, the number of GLT-1- and tdTomato-double positive cells was not affected 


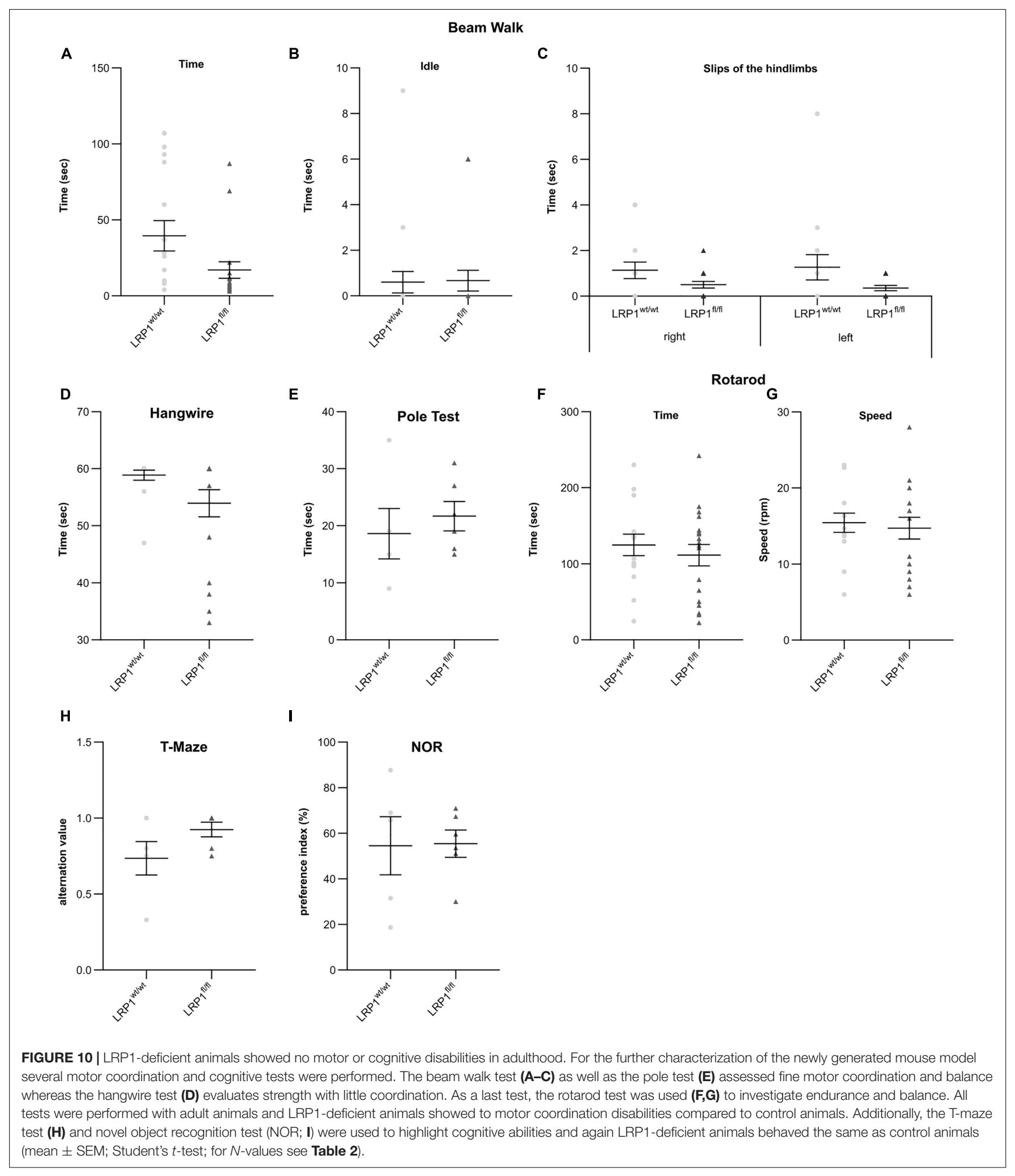

by the LRP1-knockout. Nevertheless, we observed a decreased number of GLT-1- and tdTomato-double positive cells in both conditions reaching adulthood. Benediktsson et al. (2012) observed a correlation between the number of GLT-1-positive astrocytes and neuronal activity. Therefore, we hypothesized that the observed decreased neuronal activity in the adult stage resulted in a reduced number of GLT-1- and tdTomato-positive cells in both conditions. 
Our results indicate that LRP1 is mainly essential for astroglial maturation during an early developmental phase causing a decreased maturation at this timepoint, which is compensated with ongoing development. We hypothesized that this effect might be dependent on the activation of signaling cascades as it is known that LRP1 can form complexes with other receptors on the cell surface, which induce downstream activation of signaling cascades (Seger and Krebs, 1995; Zhang and Liu, 2002; Ortega and Alcántara, 2010; Burris, 2013; Kumar et al., 2013).

Previous studies already reported that the postnatal proliferation and terminal differentiation of glial cells is dependent on cell intrinsic cues (Qian et al., 2000; Shen et al., 2006). The Ras/Raf/MEK/Erk pathway was described to be critical for the glial specification as well as for proliferation (Li et al., 2012). The activation of this signaling cascade can be induced by the complex formation of LRP1 with PDGFR $\beta$ (Boucher et al., 2002; Loukinova et al., 2002) or with trkA (Spuch et al., 2012). Therefore, we examined the phosphorylation and therewith the activation of signaling molecules Erk1/2 and Akt. Our analysis showed a significantly downregulated phosphorylation of Erk1/2 in hippocampal tissue of P21 knockout animals, which correlates to the delayed maturation observed at the same developmental stage. Therefore, we conclude that LRP1 is a regulator of the early astrocytic maturation by influencing the activation of the Ras/Raf/MEK/Erk pathway, while the maintenance of astroglial numbers seems to be not influenced by the loss of LRP1.

Based on our findings, we were interested if the delayed maturation of astrocytes had any influence on the activity of neurons as we previously observed that LRP1-depleted astrocytes negatively influenced the activity of hippocampal neurons in a model of the tripartite synapse in vitro (Romeo et al., 2021). We used c-Fos to investigate the number of activated neurons within the hippocampus and saw a significantly decreased number of c-Fos-positive cells at P21, which were not accompanied by an altered expression of NMDA or AMPA receptor subunits. This result corresponds to the delayed maturation of astrocytes at the same timepoint. Previous studies showed that the neuronal differentiation as well as neurite outgrowth is dependent on the activation of the Ras/Raf/MEK/Erk pathway induced via astrocytes as it promoted the astrocytic release of laminin (Spohr et al., 2011, 2014). We observed a decreased phosphorylation of the signaling molecule Erk1/2 involved in this signaling pathway, which besides negatively influencing the astroglial maturation also caused an altered neuronal differentiation or neurite outgrowth ultimately resulting in a decreased neuronal activity observed in our model. Further studies might highlight if the observed decreased phosphorylation of Erk1/2 is facilitated via the binding of LRP1 ligands, such as tPA or $\alpha 2$ macroglobulin, or the interaction of LRP1 with other receptors, like integrins or PDGFR.

The altered neuronal activity observed in our animal model might also result from other factors, as astrocytes are tightly associated to neuronal synapses by forming the tripartite synapse. Here, astrocytes are able to release gliotransmitters, such as GABA, ATP, and D-serine, directly influencing the activity of the pre- or postsynapse (Araque et al., 1999; Volterra and Steinhäuser, 2004; Perea et al., 2009). Another neuromodulating factor released by astrocytes is the tissue plasminogen activator (tPA) (Fernández-Monreal et al., 2004; Samson and Medcalf, 2006). It was previously reported that the inhibition of LRP1 via RAP caused a decreased uptake of tPA out of the synaptic cleft facilitated via astrocytes (Cassé et al., 2012). Thereby, astrocytes cannot buffer the potentiated NMDA receptor activity by tPA, ultimately resulting in neurotoxicity due to high levels of glutamate within the synaptic cleft (Cassé et al., 2012; Jeanneret et al., 2016).

To further characterize our new mouse model, we performed several motor coordination and cognitive tests with adult animals. However, the LRP1-deficient animals showed no disabilities or cognitive restrictions in comparison to control animals of the same age.

It is of interest to compare the outcome of the present investigation with results obtained by deleting LRP1 from cortical radial glia stem cells using the Emx1-Cre driver line (Bres et al., 2020). In that model, recombination started at E9.5 and affected both cortex and hippocampus, targeting neurogenesis as well as gliogenesis. The resulting mouse line displayed severe neurological symptoms, expanded ventricles and seizures. As a consequence, astrocytes became reactive starting at P21 and up-regulated GFAP. Unexpectedly, however, the hippocampus at P28 exhibited a reduction of c-fospositive cells, comparable to the current study. In the present approach, LRP1 was deleted from the astrocyte lineage during the postnatal period, after the phase of neurogenesis had been completed. As consequence, astrocyte maturation in the hippocampus was delayed and neuronal activity in the hippocampus appeared reduced at P14, as judged from c-fos expression. We conclude that elimination of LRP1 from the astrocyte lineage per se is not sufficient to explain the hyperactive phenotype observed in our previous study. In this our model is in agreement with a recent report where LRP1 had been removed using a GFAP-driven Cre-recombinase (Liu et al., 2017).

Summarized our results showed that the deletion of LRP1 in astrocytes resulted in a delayed maturation at an early developmental stage, accompanied by a decreased activation of signaling molecules. However, the gene expression of any stage-specific marker related to the astrocytic lineage was not influenced by the knockout induction. Though, the immunohistochemical analysis revealed a decreased number of mature astrocytes. Additionally, the neuronal activity was negatively influenced by the deletion of astroglial LRP1 at the same developmental stage. Nevertheless, the observed effects were compensated with ongoing development resulting in comparable cell numbers and no motor or cognitive deficits in adult conditional knockout mice.

\section{CONCLUSION}

In conclusion, we could identify LRP1 as a novel regulator of the early astroglial differentiation in the hippocampus by promoting 
the phosphorylation of Erk1/2 involved in the Ras/Raf/MEK/Erk signaling pathway.

\section{DATA AVAILABILITY STATEMENT}

The raw data supporting the conclusions of this article will be made available by the authors, without undue reservation.

\section{ETHICS STATEMENT}

The animal study was reviewed and approved by the State Agency for Nature, Environment and Consumer Protection Northrhine-Westphalia (Landesamt fuer Umweltschutz, Naturschutz und Verbraucherschutz; file number: 84-02.04.2016.A482). Written informed consent was obtained from the owners for the participation of their animals in this study.

\section{AUTHOR CONTRIBUTIONS}

RR performed the experiments regarding the transcardial perfusion, sample preparation, immunohistochemistry, as well as RT-PCR. RR and AF wrote and revised the manuscript. DM and $\mathrm{MM}$ performed the behavioral and cognitive tests. MM and AS revised the manuscript. All authors contributed to the article and approved the submitted version.

\section{REFERENCES}

Araque, A., Parpura, V., Sanzgiri, R. P., and Haydon, P. G. (1999). Tripartite synapses: glia, the unacknowledged partner. Trends Neurosci. 22, 208-215. doi: 10.1016/s0166-2236(98)01349-6

Auderset, L., Landowski, L. M., Foa, L., and Young, K. M. (2016). Low density lipoprotein receptor related proteins as regulators of neural stem and progenitor cell function. Stem Cells Int. 2016:2108495. doi: 10.1155/2016/210 8495

Barcelona, P. F., Ortiz, S. G., Chiabrando, G. A., and Sánchez, M. C. (2011). alpha2-Macroglobulin induces glial fibrillary acidic protein expression mediated by low-density lipoprotein receptor-related protein 1 in Müller cells. Invest. Ophthalmol. Vis. Sci. 52, 778-786. doi: 10.1167/iovs.105759

Benediktsson, A. M., Marrs, G. S., Tu, J. C., Worley, P. F., Rothstein, J. D., Bergles, D. E., et al. (2012). Neuronal activity regulates glutamate transporter dynamics in developing astrocytes. Glia 60, 175-188. doi: 10.1002/glia. 21249

Bhat, R. V., Axt, K. J., Fosnaugh, J. S., Smith, K. J., Johnson, K. A., Hill, D. E., et al. (1996). Expression of the APC tumor suppressor protein in oligodendroglia. Glia 17, 169-174. doi: 10.1002/(SICI)1098-1136(199606)17: $2<169:: A I D-G L I A 8<3.0 . C O ; 2-Y$

Bin, J. M., Harris, S. N., and Kennedy, T. E. (2016). The oligodendrocyte-specific antibody 'CC1' binds Quaking 7. J. Neurochem. 139, 181-186. doi: 10.1111/jnc. 13745

Binder, R. J., Han, D. K., and Srivastava, P. K. (2000). CD91: a receptor for heat shock protein gp96. Nat. Immunol. 1, 151-155. doi: 10.1038/77835

Boucher, P., Liu, P., Gotthardt, M., Hiesberger, T., Anderson, R. G. W., and Herz, J. (2002). Platelet-derived growth factor mediates tyrosine phosphorylation

\section{FUNDING}

We would like to thank the German Research Foundation (DFG) for their support (SPP 1757 FA 159/20-1,2 to AF, a junior research support grant to RR, and MA 5806/2-1 and MA 5806/1-2 to MM).

\section{ACKNOWLEDGMENTS}

We thank M. Sc. Kristin Glotzbach for advice concerning the manuscript and the induction of the knockout. We thank Dr. Frank Kirchhoff for support and critical comments on the manuscript. We are grateful to Dr. Magdalena Goetz for providing the GLAST-CreERT2 mouse line. We acknowledge support by the Open Access Publication funds of the RuhrUniversity Bochum.

\section{SUPPLEMENTARY MATERIAL}

The Supplementary Material for this article can be found online at: https://www.frontiersin.org/articles/10.3389/fncel. 2021.642521/full\#supplementary-material

Supplementary Figure 1 | Exemplary picture of analyzed area. The quantification of recombined and marker-positive cells occurred in the spaces between the Cornu Ammonis layers of the hippocampus. Here, the vast majority of cells were tdTomato-positive, leading to the assumption that the vast majority were astroglia.

Supplementary Figure 2 | Protein expression of GLT-1 was not altered upon knockout induction. The deletion of LRP1 from astrocytes did not cause any changes in the protein expression of GLT-1 (A,B; mean \pm SEM; two-way ANOVA with post-hoc Bonferroni test; for $N$-values see Table 2).

of the cytoplasmic domain of the low Density lipoprotein receptor-related protein in caveolae. J. Biol. Chem. 277, 15507-15513. doi: 10.1074/jbc.M20042 8200

Bres, E. E., and Faissner, A. (2019). Low density receptor-related protein 1 interactions with the extracellular matrix: more than meets the eye. Front. Cell Dev. Biol. 7:31. doi: 10.3389/fcell.2019.00031

Bres, E. E., Safina, D., Müller, J., Bedner, P., Yang, H., Helluy, X., et al. (2020). Lipoprotein receptor loss in forebrain radial glia results in neurological deficits and severe seizures. Glia 68, 2517-2549. doi: 10.1002/glia.23869

Burris, H. A. (2013). Overcoming acquired resistance to anticancer therapy: focus on the PI3K/AKT/mTOR pathway. Cancer Chemother. Pharmacol. 71, 829-842. doi: 10.1007/s00280-012-2043-3

Bushong, E. A., Martone, M. E., and Ellisman, M. H. (2004). Maturation of astrocyte morphology and the establishment of astrocyte domains during postnatal hippocampal development. Int. J. Dev. Neurosci. 22, 73-86. doi: 10. 1016/j.ijdevneu.2003.12.008

Cassé, F., Bardou, I., Danglot, L., Briens, A., Montagne, A., Parcq, J., et al. (2012). Glutamate controls tPA recycling by astrocytes, which in turn influences glutamatergic signals. J. Neurosci. 32, 5186-5199. doi: 10.1523/JNEUROSCI. 5296- 11.2012

Deacon, R. M. J., and Rawlins, J. N. P. (2006). T-maze alternation in the rodent. Nat. Protoc. 1, 7-12. doi: 10.1038/nprot.2006.2

Fernández-Monreal, M., López-Atalaya, J. P., Benchenane, K., Léveillé, F., Cacquevel, M., Plawinski, L., et al. (2004). Is tissue-type plasminogen activator a neuromodulator? Mol. Cell. Neurosci. 25, 594-601. doi: 10.1016/j.mcn.2003. 11.002

Franzdóttir, S. R., Engelen, D., Yuva-Aydemir, Y., Schmidt, I., Aho, A., and Klämbt, C. (2009). Switch in FGF signalling initiates glial differentiation in the Drosophila eye. Nature 460, 758-761. doi: 10.1038/nature08167 
Freeman, M. R. (2010). Specification and morphogenesis of astrocytes. Science 330, 774-778. doi: 10.1126/science.1190928

Fuss, B., Mallon, B., Phan, T., Ohlemeyer, C., Kirchhoff, F., Nishiyama, A., et al. (2000). Purification and analysis of in vivo-differentiated oligodendrocytes expressing the green fluorescent protein. Dev. Biol. 218, 259-274. doi: 10.1006/ dbio.1999.9574

Gordon, G. R. J., Mulligan, S. J., and MacVicar, B. A. (2007). Astrocyte control of the cerebrovasculature. Glia 55, 1214-1221. doi: 10.1002/glia.20543

Hennen, E., Czopka, T., and Faissner, A. (2011). Structurally distinct LewisX Glycans distinguish subpopulations of neural stem/progenitor cells. J. Biol. Chem. 286, 16321-16331. doi: 10.1074/jbc.M110.201095

Herrmann, J. E., Imura, T., Song, B., Qi, J., Ao, Y., Nguyen, T. K., et al. (2008). STAT3 is a critical regulator of astrogliosis and scar formation after spinal cord injury. J. Neurosci. 28, 7231-7243. doi: 10.1523/JNEUROSCI.1709-08.2008

Herz, J., Clouthier, D. E., and Hammer, R. E. (1992). LDL receptor-related protein internalizes and degrades UPA-PAI- 1 complexes and is essential for embryo implantation. Cell 71, 411-421. doi: 10.1016/0092-8674(92)90511-A

Herz, J., Clouthier, D. E., and Hammer, R. E. (1993). Correction: LDL receptorrelated protein internalizes and degrades uPA-PAI-1 complexes and is essential for embryo implantation. Cell 73:428. doi: 10.1016/0092-8674(93)90130-I

Iadecola, C., and Nedergaard, M. (2007). Glial regulation of the cerebral microvasculature. Nat. Neurosci. 10, 1369-1376. doi: 10.1038/nn2003

Jahn, H. M., Kasakow, C. V., Helfer, A., Michely, J., Verkhratsky, A., Maurer, H. H., et al. (2018). Refined protocols of tamoxifen injection for inducible DNA recombination in mouse astroglia. Sci. Rep. 8:5913. doi: 10.1038/s41598-01824085-9

Jeanneret, V., Wu, F., Merino, P., Torre, E., Diaz, A., Cheng, L., et al. (2016). Tissue-type plasminogen activator (tPA) modulates the postsynaptic response of cerebral cortical neurons to the presynaptic release of glutamate. Front. Mol. Neurosci. 9:121. doi: 10.3389/fnmol.2016.00121

Kriegstein, A., and Alvarez-Buylla, A. (2009). The glial nature of embryonic and adult neural stem cells. Annu. Rev. Neurosci. 32, 149-184. doi: 10.1146/annurev. neuro.051508.135600

Kumar, A., Rajendran, V., Sethumadhavan, R., and Purohit, R. (2013). AKT kinase pathway: a leading target in cancer research. Sci. World J. 2013:756134. doi: $10.1155 / 2013 / 756134$

Li, X., Newbern, J. M., Wu, Y., Morgan-Smith, M., Zhong, J., Charron, J., et al. (2012). MEK Is a key regulator of gliogenesis in the developing brain. Neuron 75, 1035-1050. doi: 10.1016/j.neuron.2012.08.031

Lillis, A. P., Mikhailenko, I., and Strickland, D. K. (2005). Beyond endocytosis: LRP function in cell migration, proliferation and vascular permeability. J. Thromb. Haemost. 3, 1884-1893. doi: 10.1111/j.1538-7836.2005.01371.x

Liu, C.-C., Hu, J., Zhao, N., Wang, J., Wang, N., Cirrito, J. R., et al. (2017). Astrocytic LRP1 mediates brain A $\beta$ clearance and impacts amyloid deposition. J. Neurosci. 37, 4023-4031. doi: 10.1523/JNEUROSCI.3442-16.2017

Liu, C. X., Musco, S., Lisitsina, N. M., Yaklichkin, S. Y., and Lisitsyn, N. A. (2000). Genomic organization of a new candidate tumor suppressor gene. LRP1B. Genomics 69, 271-274. doi: 10.1006/geno.2000.6331

Liu, Q., Zhang, J., Zerbinatti, C., Zhan, Y., Kolber, B. J., Herz, J., et al. (2019). Correction: lipoprotein receptor LRP1 regulates leptin signaling and energy homeostasis in the adult central nervous system. PLoS Biol. 17:e3000310. doi: 10.1371/journal.pbio.3000310

Liu, Y., Beyer, A., and Aebersold, R. (2016). On the dependency of cellular protein levels on mRNA abundance. Cell 165, 535-550. doi: 10.1016/j.cell.2016.03.014

Loukinova, E., Ranganathan, S., Kuznetsov, S., Gorlatova, N., Migliorini, M. M., Loukinov, D., et al. (2002). Platelet-derived growth factor (PDGF)-induced tyrosine phosphorylation of the low density lipoprotein receptor-related protein (LRP). Evidence for integrated co-receptor function betwenn LRP and the PDGF. J. Biol. Chem. 277, 15499-15506. doi: 10.1074/jbc.M20042 7200

Lueptow, L. M. (2017). Novel object recognition test for the investigation of learning and memory in mice. J. Vis. Exp. 30:55718. doi: 10.3791/55718

Maejima, T., Wollenweber, P., Teusner, L. U. C., Noebels, J. L., Herlitze, S., and Mark, M. D. (2013). Postnatal loss of P/Q-type channels confined to rhombiclip-derived neurons alters synaptic transmission at the parallel fiber to purkinje cell synapse and replicates genomic Cacnala mutation phenotype of ataxia and seizures in mice. J. Neurosci. 33, 5162-5174. doi: 10.1523/JNEUROSCI.544212.2013
Malatesta, P., Hack, M. A., Hartfuss, E., Kettenmann, H., Klinkert, W., Kirchhoff, F., et al. (2003). Neuronal or glial progeny: regional differences in radial glia fate. Neuron 37, 751-764. doi: 10.1016/s0896-6273(03)00116-8

Mark, M. D., Maejima, T., Kuckelsberg, D., Yoo, J. W., Hyde, R. A., Shah, V., et al. (2011). Delayed postnatal loss of P/Q-type calcium channels recapitulates the absence epilepsy, dyskinesia, and ataxia phenotypes of genomic Cacnala mutations. J. Neurosci. 31, 4311-4326. doi: 10.1523/JNEUROSCI.5342-10. 2011

Marschang, P., Brich, J., Weeber, E. J., Sweatt, J. D., Shelton, J. M., Richardson, J. A., et al. (2004). Normal development and fertility of knockout mice lacking the tumor suppressor gene LRP1b suggest functional compensation by LRP1. Mol. Cell Biol. 24, 3782-3793. doi: 10.1128/MCB.24.9.3782-3793. 2004

Mori, S., and Zhang, J. (2006). Principles of diffusion tensor imaging and its applications to basic neuroscience research. Neuron 51, 527-539. doi: 10.1016/ j.neuron.2006.08.012

Mori, T., Buffo, A., and Götz, M. (2005). The novel roles of glial cells revisited: the contribution of radial glia and astrocytes to neurogenesis. Curr. Top Dev. Biol. 69, 67-99. doi: 10.1016/S0070-2153(05)69004-7

Mori, T., Tanaka, K., Buffo, A., Wurst, W., Kühn, R., and Götz, M. (2006). Inducible gene deletion in astroglia and radial glia-a valuable tool for functional and lineage analysis. Glia 54, 21-34. doi: 10.1002/glia.20350

Muratoglu, S. C., Mikhailenko, I., Newton, C., Migliorini, M., and Strickland, D. K. (2010). Low density lipoprotein receptor-related protein 1 (LRP1) forms a signaling complex with platelet-derived growth factor receptor-beta in endosomes and regulates activation of the MAPK pathway. J. Biol. Chem. 285, 14308-14317. doi: 10.1074/jbc.M109.046672

Ortega, J. A., and Alcántara, S. (2010). BDNF/MAPK/ERK-induced BMP7 expression in the developing cerebral cortex induces premature radial glia differentiation and impairs neuronal migration. Cereb. Cortex 20, 2132-2144. doi: 10.1093/cercor/bhp275

Pekny, M., Levéen, P., Pekna, M., Eliasson, C., Berthold, C. H., Westermark, B., et al. (1995). Mice lacking glial fibrillary acidic protein display astrocytes devoid of intermediate filaments but develop and reproduce normally. EMBO J. 14, 1590-1598. doi: 10.1002/j.1460-2075.1995.tb07147.x

Pekny, M., and Pekna, M. (2004). Astrocyte intermediate filaments in CNS pathologies and regeneration. J. Pathol. 204, 428-437. doi: 10.1002/path.1645

Perea, G., Navarrete, M., and Araque, A. (2009). Tripartite synapses: astrocytes process and control synaptic information. Trends Neurosci. 32, 421-431. doi: 10.1016/j.tins.2009.05.001

Qian, X., Shen, Q., Goderie, S. K., He, W., Capela, A., Davis, A. A., et al. (2000). Timing of CNS cell generation: a programmed sequence of neuron and glial cell production from isolated murine cortical stem cells. Neuron 28, 69-80. doi: 10.1016/s0896-6273(00)00086-6

Rohlmann, A., Gotthardt, M., Willnow, T. E., Hammer, R. E., and Herz, J. (1996). Sustained somatic gene inactivation by viral transfer of Cre recombinase. Nat. Biotechnol. 14, 1562-1565. doi: 10.1038/nbt1196-1562

Romeo, R., Glotzbach, K., Scheller, A., and Faissner, A. (2021). Deletion of LRP1 from astrocytes modifies neuronal network activity in an in vitro model of the tripartite synapse. Front. Cell. Neurosci. 14:567253. doi: 10.3389/fncel.2020. 567253

Safina, D., Schlitt, F., Romeo, R., Pflanzner, T., Pietrzik, C. U., Narayanaswami, V., et al. (2016). Low-density lipoprotein receptor-related protein 1 is a novel modulator of radial glia stem cell proliferation, survival, and differentiation. Glia 64, 1363-1380. doi: 10.1002/glia.23009

Samson, A. L., and Medcalf, R. L. (2006). Tissue-type plasminogen activator: a multifaceted modulator of neurotransmission and synaptic plasticity. Neuron 50, 673-678. doi: 10.1016/j.neuron.2006.04.013

Savtchouk, I., and Volterra, A. (2018). Gliotransmission: beyond black-and-white. J. Neurosci. 38, 14-25. doi: 10.1523/JNEUROSCI.0017-17.2017

Schäfer, I., Kaisler, J., Scheller, A., Kirchhoff, F., Haghikia, A., and Faissner, A. (2019). Conditional deletion of LRP1 leads to progressive loss of recombined NG2-expressing Oligodendrocyte precursor cells in a novel mouse model. Cells 8:1550. doi: $10.3390 /$ cells 8121550

Seger, R., and Krebs, E. G. (1995). The MAPK signaling cascade. FASEB J. 9, 726-735. doi: 10.1096/fasebj.9.9.7601337

Shen, Q., Wang, Y., Dimos, J. T., Fasano, C. A., Phoenix, T. N., Lemischka, I. R., et al. (2006). The timing of cortical neurogenesis is encoded within lineages 
of individual progenitor cells. Nat. Neurosci. 9, 743-751. doi: 10.1038/nn 1694

Shinohara, M., Tachibana, M., Kanekiyo, T., and Bu, G. (2017). Role of LRP1 in the pathogenesis of Alzheimer's disease: evidence from clinical and preclinical studies. J. Lipid Res. 58, 1267-1281. doi: 10.1194/jlr.R07 5796

Sofroniew, M. V., and Vinters, H. V. (2010). Astrocytes: biology and pathology. Acta Neuropathol. 119, 7-35. doi: 10.1007/s00401-009-0619-8

Spohr, T. C. E., Dezonne, R. S., Rehen, S. K., and Gomes, F. C. A. (2011). Astrocytes treated by lysophosphatidic acid induce axonal outgrowth of cortical progenitors through extracellular matrix protein and epidermal growth factor signaling pathway. J. Neurochem. 119, 113-123. doi: 10.1111/j.1471-4159.2011. 07421.x

Spohr, T. C. E., Dezonne, R. S., Rehen, S. K., and Gomes, F. C. A. (2014). LPAprimed astrocytes induce axonal outgrowth of cortical progenitors by activating PKA signaling pathways and modulating extracellular matrix proteins. Front. Cell Neurosci. 8:296. doi: 10.3389/fncel.2014.00296

Spuch, C., Ortolano, S., and Navarro, C. (2012). LRP-1 and LRP-2 receptors function in the membrane neuron. Trafficking mechanisms and proteolytic processing in Alzheimer's disease. Front. Physiol. 3:269. doi: 10.3389/fphys.2012. 00269

Ulc, A., Zeug, A., Bauch, J., van Leeuwen, S., Kuhlmann, T., FfrenchConstant, C., et al. (2019). The guanine nucleotide exchange factor Vav3 modulates oligodendrocyte precursor differentiation and supports remyelination in white matter lesions. Glia 67, 376-392. doi: 10.1002/glia. 23548
Volterra, A., and Steinhäuser, C. (2004). Glial modulation of synaptic transmission in the hippocampus. Glia 47, 249-257. doi: 10.1002/glia. 20080

Weber, P., Metzger, D., and Chambon, P. (2001). Temporally controlled targeted somatic mutagenesis in the mouse brain. Eur. J. Neurosci. 14, 1777-1783. doi: 10.1046/j.0953-816x.2001.01803.x

Wiese, S., Karus, M., and Faissner, A. (2012). Astrocytes as a source for extracellular matrix molecules and cytokines. Front. Pharmacol. 3:120. doi: 10.3389/fphar. 2012.00120

Yong, V. W., Krekoski, C. A., Forsyth, P. A., Bell, R., and Edwards, D. R. (1998). Matrix metalloproteinases and diseases of the CNS. Trends Neurosci. 21, 75-80. doi: 10.1016/s0166-2236(97)01169-7

Zhang, W., and Liu, H. T. (2002). MAPK signal pathways in the regulation of cell proliferation in mammalian cells. Cell Res. 12, 9-18. doi: 10.1038/sj.cr.7290105

Conflict of Interest: The authors declare that the research was conducted in the absence of any commercial or financial relationships that could be construed as a potential conflict of interest.

Copyright (C) 2021 Romeo, Boden-El Mourabit, Scheller, Mark and Faissner. This is an open-access article distributed under the terms of the Creative Commons Attribution License (CC BY). The use, distribution or reproduction in other forums is permitted, provided the original author(s) and the copyright owner(s) are credited and that the original publication in this journal is cited, in accordance with accepted academic practice. No use, distribution or reproduction is permitted which does not comply with these terms. 